ECCOMAS

Proceedia
UNCECOMP 2021

$4^{\text {th }}$ ECCOMAS Thematic Conference on Uncertainty Quantification in Computational Sciences and Engineering M. Papadrakakis, V. Papadopoulos, G. Stefanou (eds.) Streamed from Athens, Greece, 28 -30 June 2021

\title{
CALIBRATION OF MATERIAL MODEL PARAMETERS USING MIXED-EFFECTS MODELS
}

\author{
Clément LABOULFIE ${ }^{1}$, Mathieu BALESDENT ${ }^{2}$, Loïc BREVAULT ${ }^{2}$, Sebastien DA \\ VEIGA $^{3}$, François-Xavier IRISARRI ${ }^{1}$, Rodolphe LE RICHE ${ }^{4}$, Jean-François MAIRE ${ }^{1}$ \\ ${ }^{1}$ DMAS, ONERA, Université Paris Saclay \\ F-92322, Châtillon FRANCE \\ e-mail: clement.laboulfie,francois-xavier.irisarri,jean-francois.maire@onera.fr
}

${ }^{2}$ DTIS, ONERA, Université Paris Saclay

F-91123, Palaiseau FRANCE

e-mail: mathieu.balesdent,loic.brevault@onera.fr

${ }^{3}$ Safran TECH

Rue des jeunes Bois, 78117 Châteaufort FRANCE

sebastien.da-veiga@ safrangroup.com

${ }^{4}$ CNRS LIMOS

Mines Saint-Étienne and UCA, 158 cours Fauriel, 42100 Saint-Étienne, FRANCE

leriche@emse.fr

\begin{abstract}
The quantification of model parameter uncertainty is a long-standing issue in model calibration. Classical techniques provide methods to handle some type of uncertainties (e.g. experimental noise or model bias). However, usual calibration techniques are not designed to take into account the variability between the different individuals. This is not a problem if the individual variability is negligible but it is an important issue if the individual variability is signifcant. The mixed-effects models provide a statistical framework to calibrate the parameters of a model taking into account the individual variability. The objective of this paper is to introduce the mixed-effects in material science. The ONERA Damage model (ODM) is considered, first with synthetic data, then with thirteen experimental strain-stress curves of a ceramic matrix composite material. The robustness of the mixed-effects approach regarding the variability and the number of specimen is investigated. Model choices such as the correlation between ODM parameters and other settings are discussed. The ability of mixed-effects models to characterize the material variability and to provide accurate estimates of the parameters associated to each specimen is illustrated.
\end{abstract}

Keywords: Model Calibration, Uncertainty Quantification, Mixed-effects models, Composite 


\section{GENERAL INTRODUCTION}

Model calibration is an active research topic which is common to all scientific domains such as hydrology [1], economics [2], biology [3] and mechanics [4]. The goal of calibration may be described as follows: for a given set of experimental observations and a model whose predictions are controlled by parameters, find the model parameters values that provide the best adequation between the model responses and the observations. Most of the time, the observations are noisy, the models may not be able to reproduce perfectly the observations (model bias), the specimens are subject to variability (due to the production process). As a consequence, the inferred model parameters are uncertain. Characterizing the uncertainty of the calibrated model parameters is essential before carrying out further analyses (uncertainty propagation, sensitivity analysis, etc.).

In the field of mechanics, for a given experiment (a tensile test for instance) and a given material, international standards impose to repeat the tests on different specimens of this material ([5] in aeronautics) to quantify the effects of material variability on the mechanical properties (e.g., the Young's modulus, the ultimate tensile strength). As a consequence, databases used to characterize materials are often composed of the results of an experiment on different specimens of the same material. Taking into account experimental variability in the calibration process is necessary when the model parameters are sensitive to them.

A large number of calibration procedures exist which are commonly divided into the frequentist $[6,7]$ and the Bayesian methods $[8,9]$. The frequentist approach consists in minimizing over the model parameters a misfit function [6] which quantifies a difference between the model output and the data. The least-square criterion and other $L_{p}$-norms $[7,10,11]$ are usual misfit criteria. In [12], other criteria such as the weighted least squares are proposed. The results of the calibration depend on the chosen criterion and on the optimization algorithm. Another type of criterion commonly used is the maximum likelihood estimator (MLE) [9, 13, 14]. For a given statistical hypothesis over the misfit (e.g., the misfit follows a Gaussian distribution), the likelihood measures how well the model output matches the data. The frequentist framework also provides methods to handle the calibration of multiple experiments assuming a single value for the input model parameters [15]. This scenario can arise if the database is composed of repetitions of a tensile test. In this case, the criterion to be minimized is a vector containing the scalar misfit criteria associated to each repetition. To solve such multi-objective optimization problem, either the problem is transformed into a single objective problem (for instance by a weighted sum of the objectives) or a multi-objective algorithm is used [16]. In the multi-objective formulation, the result of the calibration is a set of parameters values called the Pareto frontier which contains all the tradeoffs between the different misfit criteria. This set is made of the parameters which are consistent in the Pareto sense with respect to the different repetitions. In the frequentist framework, irrespectively of the number of misfit criteria, model parameters are assumed to be deterministic. However, because of the presence of experimental noises and specimens variability, the model parameters should be considered as uncertain. The frequentist framework provides methods (such as the asymptotic theory $[2,6,11]$ and bootstrap $[9,17,18])$ to quantify model parameter uncertainties but they 0are mainly dedicated to characterize the experimental noise, not the material variability.

In the Bayesian framework [6,9], the parameters are considered as random variables. The aim of the Bayesian inference is to get a description of their probability density function (PDF). 
It relies on an assumed prior density and on a likelihood function. The prior density sums up the available knowledge before calibration. The choice of the prior may be difficult and has an impact on the calibration results. As in the frequentist framework, the likelihood function expresses the goodness of fit of a given set of model parameters. The result of Bayesian inference is a PDF of the model parameters called the posterior distribution or a set of samples of it. This PDF combines information from the prior density, the likelihood and the available data $[6,8]$. Most of the time, the posterior distribution cannot be computed analytically. In case no analytical expression of the posterior distribution is available, Monte Carlo Markov Chain (MCMC) methods allow to generate samples from this posterior density $[19,20]$. The MCMCs build a random walk in the parameters space which selects samples of the posterior density[19]. The properties of the random walk ensure that the approximate posterior distribution converges to the exact posterior distribution as the number of iterations goes to infinity. Among the MCMC algorithms, one of the most popular algorithms is the Metropolis-Hastings algorithm [21, 22]. In practice, it may be challenging to define the settings of MCMC algorithms and to ensure an appropriate convergence to the exact posterior distribution.

Both frequentist and Bayesian frameworks rely on the likelihood function which expresses the goodness of fit of the model response to the data given a set of model parameters. In the material engineering literature [23, 24], both approaches make the same hypothesis towards material variability. Indeed, in the derivation of the likelihood function, it is assumed that all the specimens can be described by a unique set of model parameters. If the material variability can be neglected, this assumption is correct and allows to calibrate a single specimen. Under such an hypothesis, material model calibration has been carried out using both frequentist and Bayesian approaches. For instance, Avril et al. [23] gave examples of mechanically suited criteria to be minimized in a frequentist approach like the Finite Element Method Updating approach or the Constitutive Equation Gap Method. Within the frequentist framework again, Chongshuai et al. [25] calibrated a visco-elastic model and Solanki et al. a non-linear damage model [26]. Bayesian inference has long been applied to mechanical problems. Isenberg presented in [24] the calibration of elastic properties. Gogu et al. compared results from both frequentist and Bayesian approaches [27]. Gogu et al. later proposed the identification of elastic properties using Finite Element Model for composite materials [28]. Non-linear models can also be calibrated thanks to Bayesian inference. Liu \& Au [29] calibrated a non-linear hysteretic model, Rizzi et al. [30] proposed the identification of a finite element plasticity model and Rappel et al. calibrated visco-elastic models [31]. Rappel et al. [4] also proposed a tutorial to Bayesian inference for different mechanical models among which elastic linear, linear elasticity-perfect plasticity models and viscoelastic models.

When material variability is significant, the specimens should no longer be described by a unique set of model parameters. It is possible to calibrate individually each specimen, but the parameter vector inferred on one specimen is not necessarily consistent with the observations of the other specimens. In fact, the simple likelihood function is not designed to take into account material variability. The result of this calibration consists of a set of parameter vectors, one for each calibrated specimen. Yet, these parameter vectors are somehow related as all of them characterize the same material on which has been carried out the same experiment. Rizzi et al. [30] proposed a way to take into account material variability in the calibration process. They made the hypothesis that for all the available specimens, the model parameters vary between a lower and upper bound (defined by expert knowledge through mathematical 
constraints). This is equivalent to assuming that, for all the specimens, the model parameters are uniformly distributed between those bounds and thus can be approximated with a first order Legendre uniform polynomial chaos expansion (PCE). The objective was to find the coefficients of the PCE for each model parameter. This approach is useful as the calibration process tries to take explictly into account the material variability. However, it does not estimate the parameters of every specimen which are necessary for physical interpretations. To sum up, none of the previous methods allows to calibrate a material model parameter taking into account both experiment repetitions and material variability.

The aim of this paper is to propose a calibration method compliant with specimens repetition (defined in the following as a population) in the presence of material variability. The approach relies on mixed-effects models [32]. We seek to demonstrate the ability of the method to both describe the individual variability and to provide estimates of the individual parameters. In Section 2.1, the mixed-effects approach is presented. Section 2.3 gives two different methods to estimate the likelihood function. Then, this new methodology is applied for the first time to a simplified damage model derived from a material model for ceramic matrix composite materials (called ONERA Damage Model (ODM) [33, 34, 35]) with four parameters to be calibrated. In Section 3.3, the method is tested on virtual data to study the calibration methodology settings and the consequences of the mixed-effects. Then, in Section 3.4, thirteen individual replicate tensile experiments (that each contains a dozen to a hundred observations) are processed with the method. The specimens are made of CERASEP A400 [36, 37], a ceramic-matrix composite material.

\section{POPULATION-BASED APPROACH AND MIXED-EFFECTS MODELS}

The mixed-effects models and the population-based approaches are now described. The models are first presented under a general scope to illustrate how this point of view can help to analyze the material variability (in section 2.1 ). Then, the models are detailed and specified for the calibration of a material.

\subsection{Introduction to the population-based and the mixed-effects models}

Population-based models attempt to describe the variability of physical phenomena observed in a population of individuals. This type of approaches finds its origin in pharmacometrics $[38,39]$ where it is important to quantitatively describe interactions between drugs, diseases and patients. For instance, during a drug test, every subject is given the same amount of drug but his response heavily depends on his genome $[32,39]$. The mixed-effects notion comes from the fact that there are "fixed" effects that are shared by the entire population of individuals and "random" effects that are specific to each individual of the population. For a given model of interest, the specimen parameters (also referred as the individual parameters) can be decomposed as

$$
\text { individual parameters }=\text { fixed effects }+ \text { random effects }
$$

In a mechanical context, if the studied parameter is the Young's modulus, Eq.(1) states that the Young's modulus of each specimen is the combination of a reference value (for instance given by the producer) and of a deviation due to the production process. Depending on the relationship between the input parameters and the responses, linear [40, 41, 42] and non-linear [43] mixed-effects models have been developped.

In Figure 1, the differences between the classical and the population-based approach are highlighted. On the left of the Figure, in the classical approach, the underlying hypothesis is that 


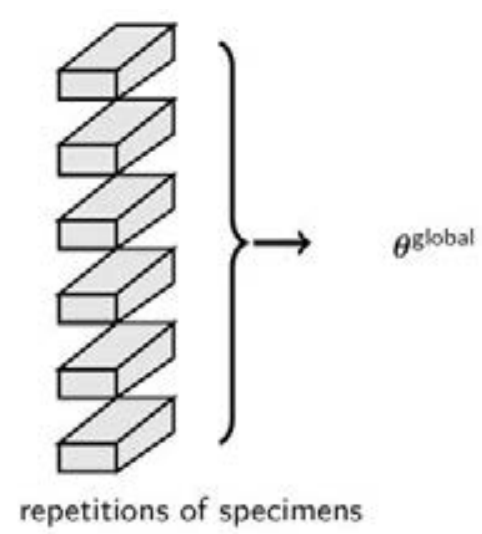

Usual methods: a single value for the parameter vector for all specimens
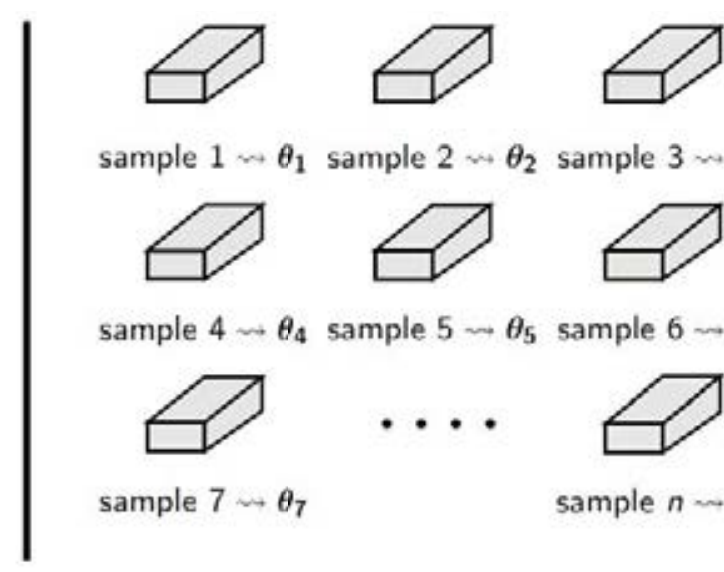

sample $1 \cdots \theta_{1}$ sample $2 \rightsquigarrow \theta_{2}$ sample $3 \rightsquigarrow \theta_{3}$
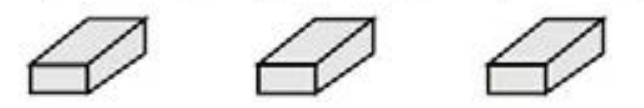

sample $4 \sim \theta_{4}$ sample $5 \sim \theta_{5}$ sample $6 \sim \theta_{6}$

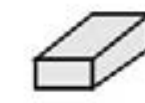

sample $7 \leadsto \theta_{7}$

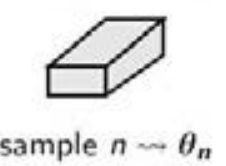

Population-based approach in the mixed-models effects framework : $\theta_{i} \sim f_{\Theta}$ and $\theta_{i} \neq \theta_{j}$

Figure 1: Comparisons of the assumptions made with respect to the individual variability in the classical approach (left) and in the population-based approach (right).

all the specimens can be described by a unique set of parameters. In the population-based approach (on the right), each sample is assigned a set of parameters. In the mixed-effects models, it is assumed that there exists an underlying probability distribution (noted $f_{\Theta}$ ) whose outcomes are the individual parameters set $\boldsymbol{\theta}_{\mathbf{1}}, \boldsymbol{\theta}_{\mathbf{2}}, \ldots, \boldsymbol{\theta}_{\boldsymbol{n}}$ with $n$ the number of individuals [32,44]. This is an improvement from the classical approach as it establishes a relation between the individual parameters set. In this framework, both the underlying probability distribution of the model parameters and the value of the individual parameters are determined. In addition, it remains possible to include other sources of uncertainty (experimental noise, model bias, etc.) on the individual parameters.

Thanks to its ability to describe individual variability, the population-based approach is used in cases where this variability is important. To the best of our knowledge, this approach has never been applied to material models to account for the variability introduced by the repetition of mechanical tests over a population of specimens.

\subsection{Formalization}

We now describe the mixed-effects approach of $[32,44]$ in the context of of tensile tests performed on different specimens. All the specimens are samples from the same material, for example $\mathrm{SiC}-\mathrm{SiC}$ composites CERASEP A400. They share the same dimensions and features. There are $n$ specimens and $i \in \llbracket 1, n \rrbracket$ is the corresponding index. The number of observations the $i^{\text {th }}$ specimen (or individual) is $N_{i}$ and $j$ stands for the index of the $j^{\text {th }}$ measure (with $j \in$ $\left.\llbracket 1, N_{i} \rrbracket\right)$. The $j^{\text {th }}$ output measure of the $i^{\text {th }}$ individual is labeled as $y_{i j}$ and $t_{i j}$ stands for the $j^{\text {th }}$ input measure of the $i^{\text {th }}$ individual. The random vector of the output measures of the $i^{\text {th }}$ individual is written $Y_{i}$ and its outcome $\mathbf{y}_{i}=\left(y_{i j}\right)_{j \in \llbracket 1, N_{i} \rrbracket}$. The set of parameters of the $i^{\text {th }}$ individual is denoted $\boldsymbol{\theta}_{\boldsymbol{i}}=\left(\boldsymbol{\theta}_{\boldsymbol{i}}^{\mathbf{1}}, \boldsymbol{\theta}_{\boldsymbol{i}}^{2}, \ldots, \boldsymbol{\theta}_{\boldsymbol{i}}^{\boldsymbol{d}}\right) \in \mathbb{R}^{d}$, with $d$ the number of model parameters to be calibrated. The model of the material is noted $\mathcal{F}(\cdot)$. PDFs will be noted by the generic letter $f$. 


\subsubsection{Mixed-effects models approach}

The mixed-effects framework $[32,44]$ assumes that there exists a probability distribution $f_{\Theta}$ whose outcomes are the individual parameters:

$$
\forall i \in \llbracket 1, n \rrbracket, \boldsymbol{\theta}_{\boldsymbol{i}} \underset{\text { i.i.d. }}{\sim} f_{\Theta}
$$

where i.i.d. stands for independent and identically distributed. Both $f_{\Theta}$ and the $\boldsymbol{\theta}_{\boldsymbol{i}}$ are unknown and the aim is to determining them. In addition, if $f_{\Theta}$ is parametric (Gaussian distribution for instance), $\Pi$ stands for its parameters and $f_{\Theta}=f_{\Theta, \Pi}$. Identifying $f_{\Theta, \Pi}$ is tantamount to determining $\boldsymbol{\Pi}$. Given $\boldsymbol{\Pi}$ and $\boldsymbol{\theta}_{\boldsymbol{i}} \sim f_{\Theta, \Pi} \forall i \in \llbracket 1, n \rrbracket$, the model output $\mathbf{y}_{\mathbf{i}}$ can be written as:

$$
\forall i \in \llbracket 1, n \rrbracket, \mathbf{y}_{\mathbf{i}} \underset{\text { i.i.d. }}{\sim} \mathcal{F}\left(\cdot, \boldsymbol{\theta}_{\boldsymbol{i}}\right)+\boldsymbol{\xi}
$$

In Eq. (3), $\boldsymbol{\xi}$ stands for the random vector of the errors. It represents the experimental noise and the model bias. Without any other hypothesis, the outcomes of $\boldsymbol{\xi}$ labeled $\left(\xi_{i j}\right)_{(i, j) \in \llbracket 1, n \rrbracket \times \llbracket 1, N_{i} \rrbracket}$ are different for each individual and for each observation. The global mixed-effects models for the $j^{\text {th }}$ output measure of the $i^{\text {th }}$ individual $y_{i j}$ reads:

$$
y_{i j}=\mathcal{F}\left(t_{i j}, \theta_{i}\right)+\xi_{i j}
$$

Classically, several additional hypotheses are assumed. First, $f_{\Theta, \Pi}$ is chosen to be a multivariate Gaussian distribution ( of dimension $d$ ):

$$
f_{\Theta, \Pi}=\mathcal{N}(\boldsymbol{\mu}, \Sigma)
$$

with $\boldsymbol{\mu} \in \mathbb{R}^{d}$ the mean vector and $\Sigma \in \mathcal{M}_{d}(\mathbb{R})$ the covariance matrix. This choice has to be made taking into account expert knowledge, experimental and physical problem characteristics. As a consequence, individual parameters can be written as follows

$$
\theta_{i}=\boldsymbol{\mu}+\mathbf{b}_{i}
$$

with $\mathbf{b}_{\mathbf{i}} \underset{\text { i.i.d. }}{\sim} \mathcal{N}(0, \Sigma)$. Comparing Eqs.(1) and (6), $\boldsymbol{\mu}$ stands for the fixed effects (the same for the whole population) and $\mathbf{b}_{i}$ the random effects (different for each individual). The second hypothesis is that for each individual and each measure, the error term is a Gaussian white noise (no bias, no correlation):

$$
\xi_{i j} \underset{\text { i.i.d. }}{\sim} \mathcal{N}\left(0, \omega_{i j}\right)
$$

with $\omega_{i j}$ the variance of the noise of the $j^{\text {th }}$ output measure of the $i^{\text {th }}$ individual. The noise further is supposed to be homoscedastic, that is to say $\omega_{i j}=\omega_{i} \forall(i, j) \in \llbracket 1, n \rrbracket \times \llbracket 1, N_{i} \rrbracket$. Finally, the vector of parameters to be calibrated is denoted $\Psi$ :

$$
\Psi=(\boldsymbol{\mu}, \quad \Sigma, \quad \Omega) \in \mathbb{R}^{n+d+\frac{d(d+1)}{2}}
$$

with $\Omega=\operatorname{diag}\left(\omega_{1}, \ldots, \omega_{n}\right)$. The mixed-effects models seek $\boldsymbol{\Psi}$ and gives an estimate of the individual parameters $\left(\boldsymbol{\theta}_{\boldsymbol{i}}\right)_{i \in \llbracket 1, n \rrbracket}$. 


\subsubsection{Likelihood of mixed-effects models}

The calibration is often achieved by maximizing the likelihood of $\Psi, \mathcal{L}(\Psi)=f\left(\mathbf{y}_{1}, \ldots, \mathbf{y}_{n} \mid \Psi\right)$, even if other methods can be found to estimate $\Psi$ [45].

The first step consists in writing the PDF of ouput measurements for a given set of individual parameters and error term. Combining the Eqs.(4) and (7), this density is expressed as:

$$
f\left(\mathbf{y}_{i} \mid \boldsymbol{\theta}_{\boldsymbol{i}}, \omega_{i}\right)=\frac{1}{\left(\omega_{i} \sqrt{2 \pi}\right)^{N_{i}}} \prod_{j=1}^{N_{i}} e^{-\frac{1}{2}\left(\frac{y_{i j}-\mathcal{F}\left(t_{i j}, \boldsymbol{\theta}_{\boldsymbol{i}}\right)}{\omega_{i}}\right)^{2}}
$$

The individual parameters are distributed according to a Gaussian PDF (Eq.(5)). For a given $\Psi$, their density can be written as follows:

$$
f\left(\boldsymbol{\theta}_{\boldsymbol{i}} \mid \Psi\right)=\frac{1}{\sqrt{|\Sigma|\left(2 \pi^{d}\right)}} e^{-\frac{1}{2}\left(\boldsymbol{\theta}_{\boldsymbol{i}}-\boldsymbol{\mu}\right)^{T} \Sigma^{-1}\left(\boldsymbol{\theta}_{\boldsymbol{i}}-\boldsymbol{\mu}\right)}
$$

Following the use of the conditional probability rule, the PDF of the individual parameters $\boldsymbol{\theta}_{\boldsymbol{i}}$ and model output $\mathbf{y}_{i}$ for a given $\boldsymbol{\Psi}$, that is to say $f\left(\mathbf{y}_{i}, \boldsymbol{\theta}_{\boldsymbol{i}} \mid \Psi\right)$, is:

$$
f\left(\mathbf{y}_{i}, \boldsymbol{\theta}_{\boldsymbol{i}} \mid \boldsymbol{\Psi}\right)=f\left(\mathbf{y}_{i} \mid \boldsymbol{\theta}_{\boldsymbol{i}}, \boldsymbol{\Psi}\right) f\left(\boldsymbol{\theta}_{\boldsymbol{i}} \mid \boldsymbol{\Psi}\right)
$$

Using Eqs.(8), (9) and (10), $f\left(\mathbf{y}_{i}, \boldsymbol{\theta}_{\boldsymbol{i}} \mid \Psi\right)$ can be written as :

$$
f\left(\mathbf{y}_{i}, \boldsymbol{\theta}_{\boldsymbol{i}} \mid \boldsymbol{\Psi}\right)=\frac{1}{\omega_{i}^{N_{i}} \sqrt{|\Sigma|(2 \pi)^{N_{i}+d}}} e^{-\frac{1}{2}\left(\left(\boldsymbol{\theta}_{\boldsymbol{i}}-\boldsymbol{\mu}\right)^{T} \Sigma^{-1}\left(\boldsymbol{\theta}_{\boldsymbol{i}}-\boldsymbol{\mu}\right)+\sum_{j=1}^{N_{i}}\left(\frac{y_{i j}-\mathcal{F}\left(t_{i j}, \boldsymbol{\theta}_{\boldsymbol{i}}\right)}{\omega_{i}}\right)^{2}\right)}
$$

Because the $\boldsymbol{\theta}_{\boldsymbol{i}}$ 's are not observed, The likelihood of the $i^{\text {th }}$ individual $\mathcal{L}_{i}(\boldsymbol{\Psi})$ is the integral of $f\left(\mathbf{y}_{i}, \boldsymbol{\theta}_{\boldsymbol{i}} \mid \Psi\right)$ with respect to all possible $\boldsymbol{\theta}_{\boldsymbol{i}}$ over $\mathbb{R}^{d}$ :

$$
\mathcal{L}_{i}(\Psi)=\int_{\mathbb{R}^{d}} f\left(\mathbf{y}_{i}, \boldsymbol{\theta}_{i} \mid \Psi\right) \mathrm{d} \boldsymbol{\theta}_{\boldsymbol{i}}
$$

Under the assumption of independent individuals, the likelihood of $\Psi$ reads as the product of all the individual likelihoods,

$$
\mathcal{L}(\Psi)=\prod_{i=1}^{n} \mathcal{L}_{i}(\Psi)
$$

The maximum likelihood estimator $\hat{\Psi}$ is obtained as the result of the following maximization problem over the set of all the possible parameters $\Xi$ :

$$
\hat{\Psi}=\underset{\Psi \in \Xi}{\arg \max } \mathcal{L}(\Psi)
$$

In practice, the log-likelihood is computed to ease numerical optimization.

Mixed-effects models approach consists in calibrating a (multivariate) probability distribution. To do so, a minimum number of samples is required. However, in the material field, the number of repetitions of a given experiment is small (in the order of 10 for some tests, see [5] for tensile tests). This has to be confronted with the number of parameters to be calibrated which is here in $\mathcal{O}\left(n+d^{2}\right)$. As a consequence, the chosen modeling must be consistent with the available number of repetitions. 


\subsection{Computing the likelihood}

The evaluation of the likelihood function requires to compute the individual likelihoods, which imply to estimate the multi-dimensional integrals of Eq.(12). A fundamental method to compute the integral is the Monte-Carlo method [46]. To compute the integral of a function $g(\cdot)$ with respect to any density $f$, the Monte-Carlo method works as follows: samples are generated with respect to the PDF $f$ (potentially with MCMC methods); the integral is approximated as the empirical mean of the function $g(\cdot)$ calculated at each sample. The main issue is that the sampling density $f\left(\boldsymbol{\theta}_{\boldsymbol{i}} \mid \Psi\right)$ does not necessarily generate model parameters that result in a proper adequation of the model responses and the observations. Therefore, the likehood function often collapses to 0 . In order to generate model parameters that better suit the observations, an importance sampling scheme (written MCMC-IS) [32, 47] is implemented and described next. It will be compared to another usual methods to compute multidimensional integrals, the Laplace approximation [48]. This approach is based on an approximation of the integrand. It does not involve any sampling technique but requires to perform an auxiliary minimization.

\subsubsection{Computation of the likelihood by importance sampling}

Importance sampling scheme belongs to the same family of integration schemes as the classical Monte-Carlo [9]. An auxiliary PDF is used to generate samples in place of the initial density function. The idea is to generate model parameters associated to the model responses that are more consistent with the available observations. As a result, the likelihood function does not collapse to 0, a necessary condition for its maximization. The integral of Eq.(12) is rewritten as follows:

$$
\int_{\mathbb{R}^{d}} f\left(\mathbf{y}_{i} \mid \boldsymbol{\theta}_{\boldsymbol{i}}, \boldsymbol{\Psi}\right) f\left(\boldsymbol{\theta}_{\boldsymbol{i}} \mid \boldsymbol{\Psi}\right) \mathrm{d} \boldsymbol{\theta}_{\boldsymbol{i}}=\int_{\mathbb{R}^{d}} f\left(\mathbf{y}_{i} \mid \boldsymbol{\theta}_{\boldsymbol{i}}, \boldsymbol{\Psi}\right) \frac{f\left(\boldsymbol{\theta}_{\boldsymbol{i}} \mid \boldsymbol{\Psi}\right)}{\pi_{i}\left(\boldsymbol{\theta}_{\boldsymbol{i}} \mid \boldsymbol{\Psi}\right)} \pi_{i}\left(\boldsymbol{\theta}_{\boldsymbol{i}} \mid \boldsymbol{\Psi}\right) \mathrm{d} \boldsymbol{\theta}_{\boldsymbol{i}}
$$

with $\pi_{i}\left(\boldsymbol{\theta}_{\boldsymbol{i}} \mid \Psi\right)$ the importance sampling density. The chosen importance sampling density is $\pi_{i}\left(\boldsymbol{\theta}_{\boldsymbol{i}} \mid \Psi\right)=f\left(\boldsymbol{\theta}_{\boldsymbol{i}} \mid \mathbf{y}_{i}, \boldsymbol{\Psi}\right)$ which allows to generate model parameters conditioned on both $\Psi$ and the available observations $\mathbf{y}_{i}$. To compute the integral in Eq.(15), it is necessary to generate samples from $\pi_{i}\left(\boldsymbol{\theta}_{\boldsymbol{i}} \mid \Psi\right)$ which can be done with MCMC methods since this density is known up to a normalization constant (with respect to $\boldsymbol{\theta}_{\boldsymbol{i}}$ ). Indeed, the conditional probability rule gives

$$
f\left(\boldsymbol{\theta}_{\boldsymbol{i}} \mid \mathbf{y}_{i}, \Psi\right)=\frac{f\left(\mathbf{y}_{i}, \boldsymbol{\theta}_{\boldsymbol{i}} \mid \Psi\right)}{f\left(\mathbf{y}_{i} \mid \Psi\right)}
$$

and the numerator is known from Eq.(11). It can be noticed that the denominator which appear in Eq.(16) is in fact the individual likelihood $\mathcal{L}_{i}(\Psi)$ defined in Eq.(12). Computing the integral requires to evaluate $f\left(\mathbf{y}_{i} \mid \boldsymbol{\theta}_{\boldsymbol{i}}, \boldsymbol{\Psi}\right)$ (which is known in closed form with Eq.(8)) and the importance sampling ratio $\frac{f\left(\boldsymbol{\theta}_{i} \mid \boldsymbol{\Psi}\right)}{\pi_{i}\left(\boldsymbol{\theta}_{i} \mid \Psi\right)}$ (which demands to evaluate $f\left(\boldsymbol{\theta}_{i} \mid \mathbf{y}_{i}, \Psi\right)$ ). Samples can be generated thanks to MCMC technics but it remains necessary to evaluate the PDF value $f\left(\boldsymbol{\theta}_{\boldsymbol{i}} \mid \mathbf{y}_{i}, \Psi\right)$. To provide an estimation of $f\left(\boldsymbol{\theta}_{\boldsymbol{i}} \mid \mathbf{y}_{i}, \boldsymbol{\Psi}\right)$, it is possible to use Kernel Density Estimation methods [49]. However, with such methods, the accuracy of the PDF estimation decreases when the dimension of $\boldsymbol{\theta}_{\boldsymbol{i}}$ increases. Therefore, it is instead decided to approximate this density by

$$
\pi_{i}\left(\boldsymbol{\theta}_{\boldsymbol{i}} \mid \boldsymbol{\Psi}\right)=\mathcal{N}\left(m\left(\boldsymbol{\theta}_{\boldsymbol{i}}, \mathbf{y}_{i}, \boldsymbol{\Psi}\right), C^{2}\left(\boldsymbol{\theta}_{\boldsymbol{i}}, \mathbf{y}_{i}, \boldsymbol{\Psi}\right)\right)
$$

with $m\left(\boldsymbol{\theta}_{\boldsymbol{i}}, \mathbf{y}_{i}, \boldsymbol{\Psi}\right)=\mathbb{E}\left(\boldsymbol{\theta}_{\boldsymbol{i}} \mid \mathbf{y}_{i}, \boldsymbol{\Psi}\right)$ the empirical mean of the MCMC samples of $f\left(\boldsymbol{\theta}_{\boldsymbol{i}} \mid \mathbf{y}_{i}, \boldsymbol{\Psi}\right)$ and $C^{2}\left(\boldsymbol{\theta}_{\boldsymbol{i}}, \mathbf{y}_{i}, \Psi\right)=\mathbb{V}\left(\boldsymbol{\theta}_{\boldsymbol{i}} \mid \mathbf{y}_{i}, \Psi\right)$, the empirical covariance matrix with only diagonal terms in 
$\mathcal{M}_{d}(\mathbb{R})$. Finally, for $M$ i.i.d. samples generated with respect to $\pi_{i}\left(\boldsymbol{\theta}_{\boldsymbol{i}} \mid \Psi\right)$ as defined in Eq.(17) (labeled $\widetilde{\boldsymbol{\theta}}_{i}^{k}, k \in \llbracket 1, M \rrbracket$ ), the integral of Eq.(15) is approximated as follows:

$$
\int_{\mathbb{R}^{d}} f\left(\mathbf{y}_{i} \mid \boldsymbol{\theta}_{i}, \Psi\right) f\left(\boldsymbol{\theta}_{\boldsymbol{i}} \mid \Psi\right) \mathrm{d} \boldsymbol{\theta}_{\boldsymbol{i}} \approx \frac{1}{M} \sum_{k=1}^{M} f\left(\mathbf{y}_{i} \mid \widetilde{\boldsymbol{\theta}}_{i}^{k}, \Psi\right) \frac{f\left(\widetilde{\boldsymbol{\theta}}_{i}^{k} \mid \Psi\right)}{\pi_{i}\left(\widetilde{\boldsymbol{\theta}}_{i}^{k} \mid \Psi\right)}
$$

This computation is carried out for each individual likelihood (typically the likelihood associated to each specimen). The most computationally demanding part of the likelihood estimation is the generation of MCMC samples which requires thousands of repeated material model evaluations.

\subsubsection{Computation of the likelihood through the Laplace approximation}

The Laplace approximation [50] applies to integrals of the type

$$
A=\int_{\mathbb{R}^{d}} e^{h(\mathbf{x})} \mathrm{d} \mathbf{x}
$$

with $h(\cdot)$ a function which complies with some constraints:

1. $h(\cdot)$ admits a global maximum $\mathbf{x}_{0}$ that belongs to the integration interval,

2. $h(\cdot)$ is a twice-differentiable function,

3. its Hessian matrix computed at $\mathbf{x}=\mathbf{x}_{\mathbf{0}}$ is a symmetric definite negative matrix.

The main idea is to state that only points close to $\mathrm{x}_{0}$ significantly contribute to the integral. The different calculations that allow to establish the equations of the Laplace approximation are presented below. The Taylor expansion of $h(\cdot)$ at $\mathbf{x}_{0}$ can be written as :

$$
h(\mathbf{x})=h\left(\mathbf{x}_{0}\right)+\left(\mathbf{x}-\mathbf{x}_{0}\right)^{T} \nabla h\left(\mathbf{x}_{0}\right)+\frac{1}{2}\left(\mathbf{x}-\mathbf{x}_{0}\right)^{T} \mathcal{H}(h)\left(\mathbf{x}_{0}\right)\left(\mathbf{x}-\mathbf{x}_{0}\right)+o\left(\left\|\mathbf{x}-\mathbf{x}_{0}\right\|^{2}\right)
$$

with:

- $\nabla h\left(\mathbf{x}_{0}\right)=\left(\frac{\partial h}{\partial \mathbf{x}_{i}}\left(\mathbf{x}_{0}\right)\right)_{i \in \llbracket 1, d \rrbracket}$ the gradient vector of $h(\cdot)$ at $\mathbf{x}_{0}$,

- $\mathcal{H}(h)\left(\mathbf{x}_{0}\right)=\left(\frac{\partial^{2} h}{\partial \mathbf{x}_{i} \partial \mathbf{x}_{j}}\left(\mathbf{x}_{0}\right)\right)_{(i, j) \in \llbracket 1, d \rrbracket^{2}}$ the Hessian matrix of $h(\cdot)$ at $\mathbf{x}_{0}$.

As $\mathbf{x}_{0}$ is the global maximum, the gradient vanishes and the substitution of $h(\mathbf{x})$ in Eq.(2.3.2) by its approximation determined in Eq.(18) gives:

$$
\int_{\mathbb{R}^{d}} e^{h(\mathbf{x})} \mathrm{d} \mathbf{x} \approx \int_{\mathbb{R}^{d}} e^{h\left(\mathbf{x}_{0}\right)+\frac{1}{2}\left(\mathbf{x}-\mathbf{x}_{0}\right)^{T} \mathcal{H}(h)\left(\mathbf{x}_{0}\right)\left(\mathbf{x}-\mathbf{x}_{0}\right)} \mathrm{d} \mathbf{x}=e^{h\left(\mathbf{x}_{0}\right)} \int_{\mathbb{R}^{d}} e^{\frac{1}{2}\left(\mathbf{x}-\mathbf{x}_{0}\right)^{T} \mathcal{H}(h)\left(\mathbf{x}_{0}\right)\left(\mathbf{x}-\mathbf{x}_{0}\right)} \mathrm{d} \mathbf{x}
$$

$\mathcal{H}(h)\left(\mathbf{x}_{0}\right)$ is symmetric definite negative so $-\mathcal{H}(h)\left(\mathbf{x}_{0}\right)$ is symmetric definite positive. As a result, in Eq.(19), the integrand is a Gaussian PDF of mean $\mathbf{x}_{0}$ et and covariance matrix $-\mathcal{H}(h)\left(\mathbf{x}_{0}\right)^{-1}$. As a PDF always integrates to 1 ,

$$
\int_{\mathbb{R}^{d}} e^{-\frac{1}{2}\left(\mathbf{x}-\mathbf{x}_{0}\right)^{T} M^{-1}\left(\mathbf{x}-\mathbf{x}_{0}\right)} \mathrm{d} \mathbf{x}=(2 \pi)^{\frac{d}{2}} \sqrt{|M|}
$$


Applying Eqs.(19) and (20), the Laplace approximation of $A$ is obtained as

$$
A \approx e^{\left.h\left(\mathbf{x}_{0}\right)\right)} \frac{(2 \pi)^{\frac{d}{2}}}{\sqrt{\left|-\mathcal{H}(h)\left(\mathbf{x}_{0}\right)\right|}}
$$

For a given $\Psi=(\boldsymbol{\mu}, \Sigma, \Omega)$, the individual likelihood reads as [48]

$$
\mathcal{L}_{i}(\Psi)=\int_{\mathbb{R}^{d}} f\left(\mathbf{y}_{i} \mid \boldsymbol{\theta}_{\boldsymbol{i}}, \Psi\right) f\left(\boldsymbol{\theta}_{\boldsymbol{i}} \mid \Psi\right) \mathrm{d} \boldsymbol{\theta}_{\boldsymbol{i}}=\int_{\mathbb{R}^{d}} \frac{1}{\left(\omega_{i} \sqrt{2 \pi}\right)^{N_{i}} \sqrt{|\Sigma|\left(2 \pi^{d}\right)}} e^{-\frac{g_{i}\left(\boldsymbol{\mu}, \Delta_{i}, \mathbf{y}_{i}, \mathbf{b}_{i}\right)}{2 \omega_{i}^{2}}} \mathrm{~d} \mathbf{b}_{i}
$$

with $\boldsymbol{\theta}_{\boldsymbol{i}}=\boldsymbol{\mu}+\mathbf{b}_{i}$ where $\boldsymbol{\mu}$ stands for the mean (i.e. the fixed effects) and $\mathbf{b}_{i}$ are the individual deviations (i.e. the random effects). $\Delta_{i}$ is the result of the Cholesky decomposition of $\omega_{i}^{2} \Sigma^{-1}$ (so $\omega_{i}^{2} \Sigma^{-1}=\Delta_{i} \Delta_{i}^{T}$ ). The function $g_{i}(\cdot)$ is defined by

$$
g_{i}\left(\boldsymbol{\mu}, \Delta_{i}, \mathbf{y}_{i}, \mathbf{b}_{i}\right)=\left\|\mathbf{y}_{i}-\mathcal{F}\left(\mathbf{t}_{i}, \boldsymbol{\mu}+\mathbf{b}_{i}\right)\right\|^{2}+\left\|\Delta_{i} \mathbf{b}_{i}\right\|^{2}
$$

The Laplace method is applied in two steps:

1. Search for the individual parameters (or rather the deviations), $\hat{\mathbf{b}}_{i}$, minimizing $g_{i}(\cdot)(23)$,

2. Computation of the Laplace approximation with formula in Eq.(21).

The Hessian matrix of $g_{i}(\cdot)$ at $\hat{\mathbf{b}}_{\mathbf{i}}$ is [48]:

$$
\mathcal{H}\left(g_{i}\right)\left(\hat{\mathbf{b}}_{i}\right)=\frac{\partial^{2} \mathcal{F}\left(\boldsymbol{\mu}, \mathbf{y}_{i}, \hat{\mathbf{b}}_{i}\right)}{\partial \mathbf{b}_{i} \partial \mathbf{b}_{i}^{T}}\left(\mathbf{y}_{i}-\mathcal{F}\left(\boldsymbol{\mu}, \mathbf{y}_{i}, \hat{\mathbf{b}}_{i}\right)\right)+\frac{\partial \mathcal{F}\left(\boldsymbol{\mu}, \mathbf{y}_{i}, \hat{\mathbf{b}}_{i}\right)^{T}}{\partial \mathbf{b}_{i}} \frac{\partial \mathcal{F}\left(\boldsymbol{\mu}, \mathbf{y}_{i}, \hat{\mathbf{b}}_{i}\right)}{\partial \mathbf{b}_{i}}+\Delta_{i}^{T} \Delta_{i}
$$

In practice, $\frac{\partial^{2} \mathcal{F}\left(\boldsymbol{\mu}, \mathbf{y}_{i}, \hat{\mathbf{b}}_{i}\right)}{\partial \mathbf{b}_{i} \partial \mathbf{b}_{i}^{T}}\left(\mathbf{y}_{i}-\mathcal{F}\left(\boldsymbol{\mu}, \mathbf{y}_{i}, \hat{\mathbf{b}}_{i}\right)\right)$ can be neglected if the model $\mathcal{F}(\cdot)$ is close enough to the experiment $\mathbf{y}_{i}[51]$. The term $\frac{\partial \mathcal{F}\left(\mu, \mathbf{y}_{i}, \hat{\mathbf{b}}_{i}\right)}{\partial \mathbf{b}_{i}}$ is evaluated using a finite difference scheme. As a result, the negative log-likelihood is finally expressed as:

$$
\begin{aligned}
-\ln (\mathcal{L}(\Psi)) & =-\sum_{i=1}^{n} \ln \left(\mathcal{L}_{i}(\boldsymbol{\Psi})\right)=-\sum_{i=1}^{n} \ln \left(\int_{\mathbb{R}^{d}} f\left(\mathbf{y}_{i} \mid \boldsymbol{\theta}_{\boldsymbol{i}}, \boldsymbol{\Psi}\right) f\left(\boldsymbol{\theta}_{\boldsymbol{i}} \mid \boldsymbol{\Psi}\right) \mathrm{d} \boldsymbol{\theta}_{\boldsymbol{i}}\right) \\
& \approx \frac{n}{2} \ln (|\Sigma|)+\sum_{i=1}^{n}\left(\frac{1}{2} \ln \left(-\left|\mathcal{H}\left(g_{i}\right)\left(\hat{\mathbf{b}}_{i}\right)\right|\right)+\frac{g_{i}\left(\boldsymbol{\mu}, \Delta_{i}, \mathbf{y}_{i}, \hat{\mathbf{b}}_{i}\right)}{2 \omega_{i}^{2}}+N_{i} \ln \left(\omega_{i} \sqrt{2 \pi}\right)\right)
\end{aligned}
$$

In both approaches (MCMC-IS and Laplace), the computation of the different individual likelihoods is independent. As a result, to reduce the computational time, the calculation of the individual likehoods is performed in parallel.

\subsection{Maximizing the likelihood}

Now that the expression of the likelihood for a mixed-effects model has been established, the model parameters can be estimated by maximizing it, or equivalently (although numerically better conditioned), by minimizing minus the log-likelihood,

$$
\hat{\boldsymbol{\Psi}}=\underset{\boldsymbol{\Psi} \in \Xi}{\arg \min }-\ln (\mathcal{L}(\Psi))
$$


Remember that computing the likelihood is numerically challenging as it is necessary to estimate a multi-dimensional integral with respect to the unobserved variables Eq.(12). To solve the minimization problem (Eq. (26)), the first possibility is to rely on gradient-based algorithms which need either to compute the gradient or an approximation of the gradient of the objective function. Here, the gradient of $-\ln (\mathcal{L}(\Psi))$ with respect to $\Psi$ is difficult to compute analytically. The application of classical differentiation formulas shows that it is necessary to differentiate the individual likelihoods which is a tricky task as it requires to differentiate the integral of Eq. (12). Indeed, differentiating the integrals is difficult as both the integrand and the integration variable depend of $\Psi$ via Eq. (2). A second possibility is to use gradient-free optimization algorithms such as evolutionary algorithms but they tend to require too many likelihood estimations. As a result, neither gradient-based algorithms nor evolutionnary algorithms are used in this paper to solve the problem defined in Eq.(26).

Others methods based on likelihood maximization include the Stochastic Approximation Expectation Maximization algorithm (SAEM) [47]. The algorithm is an adaptation of the classical Expectation Maximization algorithm which uses a stochastic approximation of the likelihood [52]. The algorithm builds a sequence of estimate of $\Psi,\left(\Psi_{k}\right)_{k \in \mathbb{N}}$ which converges to the exact optimal value of $\Psi$ under regularity assumptions on the likelihood [47]. This algorithm is implemented for instance in the toolbox SAEMIX [53].

In the current work, to speed-up the convergence of the optimization, a Bayesian optimization scheme is chosen [54]. Bayesian optimization works as follows. The search starts by computing the objective function over a design of experiment (DoE) a latin hypercube sampling [55] for instance. Next, a surrogate model of the objective function is constructed with a Gaussian process [56]. The Gaussian process allows the defnition of an infill criterion (here the expected improvement [57]) which is maximized in the search space $\Xi$ to determine the location where the next exact likelihood function should be evaluated. The procedure is repeated until a stopping criterion is met, here a maximum number of likelihood estimations.

\subsection{Estimating the individual parameters}

The mixed-effects models approach allows to infer the individual parameters. With the two methods (Laplace approximation and importance sampling scheme) chosen to compute the likelihood, individual parameters are by-product of the likelihood function calculation at $\hat{\Psi}$. In the importance sampling scheme, individual parameters are predicted as the mean of the PDF $\pi_{i}\left(\boldsymbol{\theta}_{\boldsymbol{i}} \mid \Psi\right)$, i.e., the empirical mean of $\boldsymbol{\theta}_{\boldsymbol{i}} \mid \mathbf{y}_{i}, \boldsymbol{\Psi}$. Note in passing that MCMC samples can be used to propagate uncertainties through the model. With the Laplace approximation, individual parameters are computed through the minimization of function $g_{i}(\cdot)$ at $\hat{\boldsymbol{\Psi}}$ and $\hat{\boldsymbol{\theta}}_{\boldsymbol{i}}=\boldsymbol{\mu}+\hat{\mathbf{b}}_{i}$. These individual parameters can be interpreted as the mode of $f\left(\boldsymbol{\theta}_{i} \mid \mathbf{y}_{i}, \hat{\Psi}\right)$.

\section{APPLICATIONS}

In this section, the mixed-effects methodology is applied to the calibration of amaterial model of ceramic-matrix composite materials called the ONERA Damage Model (ODM) and presented in $[33,34,35]$. The ODM model is presented in Section 3.1. In Section ??, the choicesregarding the statistical model. In Section 3.3, the approach is tested on synthetic experimental data in order to carry out different sensitivity analyses of the method. Eventually, in Section 3.4, real experimental data are calibrated. 


\subsection{The ONERA Damage Model}

A simplified uni-axial version of the ODM model is now presented. The stress (in MPa) will be labeled $\sigma$ and the strain (without units) $\varepsilon$. The strain-stress relation is

$$
\sigma(t)=E^{\mathrm{eff}}\left(d_{s}(t)\right) \varepsilon(t)-E_{0} \varepsilon_{r}(t)
$$

with $E_{0}$ the Young's modulus, $E^{\text {eff }}$ the effective Young's modulus which takes into account the loss in stiffness of the material caused by the damage $d_{s}, \varepsilon_{r}$ is the residual strain and $t$ the time. The residual strain is the strain left in the absence of load. The effective Young's modulus reads

$$
E^{\mathrm{eff}}\left(d_{s}(t)\right)=\frac{E_{0}}{1+\eta(t) d_{s}(t)}
$$

The damage desactivation index, $\eta$, represents the fact that damage does not impact the stiffness for compressive loads. It is given by $\eta(t)=h(\varepsilon(t))$ with $h$ the Heaviside function. The damage $d_{s}$ is computed from the thermodynamical force $y$. The damage equations are the following

$$
\left\{\begin{array}{l}
y(t)=\frac{1}{2} E_{0}\langle\varepsilon(t)\rangle_{+}^{2} \\
g_{s}(y(t))=\frac{\sqrt{y_{\max }(t)}-\sqrt{y_{0 s}}}{\sqrt{y_{c s}}} \text { with } y_{\max }(t)=\sup _{\tau \in[0, t]} y(\tau) \\
d_{s}(t)=d_{c}\left(1-e^{-\left\langle g_{s}(y(t))\right\rangle_{+}^{p}}\right)
\end{array}\right.
$$

$\langle x\rangle_{+}$stands for the positive part of $x$ (i.e. 0 if $x<0$ and $x$ if $x>0$ ). Eq.(29) defines the thermodynamical driving force. The positive part means that damage is created only under tensile stress. In Eq.(30), the parameter $y_{0 s}$ is the damage threshold, $y_{c s}$ the damage evolution celerity, and $p$ a shape parameter called the damage evolution exponent. The damage threshold indicates the beginning of damage. Indeed, in Eqs.(29) and (30), for thermodynamical forces smaller than $y_{0}^{s}$, the damage does not increase and the model sums up to $\sigma=E_{0} \varepsilon$ using Eq.(28). To this damage threshold can be associated a strain damage threshold given by $\varepsilon_{0}^{s}=\sqrt{\frac{2 y_{0} s}{E_{0}}}$. The parameter $d_{c}$ stands for the damage saturation. The damage evolution exponent controls the regularity of the transition between the linear and non linear parts of the curve. If $p>1$, the derivative of the stress with respect to the strain (known as the tangent matrix), $\frac{\partial \sigma}{\partial \varepsilon}$, is continuous on the whole curve. However, if $p<1$, the tangent matrix is not continuous at $\varepsilon=\varepsilon_{s}^{0}$ and it can create numerical issues in finite element calculations. Nevertheless, contrary to the case $p>1$, the damage threshold is much easier to calibrate if $p<1$ as it is directly related to a kink on strain-stress curves.

The residual strain evolves according to the following equation:

$$
\frac{\partial \varepsilon_{r}}{\partial t}=\chi \eta \frac{\partial d_{s}}{\partial t}\left(\frac{E^{e f f}}{E_{0}}\right)^{2} \varepsilon
$$

In Eq.(32), only $\chi$ has to be identified. The model parameters are summed up in Table 1.

\subsection{Settings of the statistical model}

We now seek to apply the mixed-effects models methodology on strain-stress curves which can be modeled by the ODM model. The first step consists in applying the methodology to virtual data to investigate its ability to calibrate stress-strain curves and describe the material 
Table 1: ODM Model parameters.

\begin{tabular}{|c|c|c|c|c|c|}
\hline $\begin{array}{c}\text { Young's } \\
\text { modulus }\end{array}$ & $\begin{array}{c}\text { damage } \\
\text { threshold }\end{array}$ & $\begin{array}{c}\text { damage evolu- } \\
\text { tion celerity }\end{array}$ & $\begin{array}{c}\text { damage } \\
\text { saturation }\end{array}$ & $\begin{array}{c}\text { damage evolu- } \\
\text { tion exponent }\end{array}$ & $\begin{array}{c}\text { residual } \\
\text { strain }\end{array}$ \\
\hline$E_{0}$ & $y_{0 s}$ & $y_{c s}$ & $d_{c}$ & $p$ & $\chi$ \\
\hline
\end{tabular}

variability. Since the data is generated directly from the model, one knows the exact model parameters distribution and the individual parameters values, and we can confront them to the calibration results. It is also possible to evaluate the robustness of the calibration technique to the number of available individuals. More formally, for a given mean $\boldsymbol{\mu}$ and covariance matrix $\Sigma$ (this set of parameters will be denoted $\Psi_{\text {exact }}$ ), it is possible to sample the individual parameters which are labeled $\boldsymbol{\theta}_{\mathbf{i} \text {,exact }}$. For each of these individual parameters, the ODM model outputs $\sigma_{i, \text { calc }}$ are computed, which stand for the different specimens. Using the mixed-effects approach, a calibrated distribution characterized by the estimated distribution parameters (labeled $\hat{\Psi}$ ) and the corresponding individual parameters (denoted $\hat{\boldsymbol{\theta}}_{\boldsymbol{i}}$ ) is obtained. One objective consists in checking that the calibrated distribution is consistent with the exact distribution, the virtual data and the exact individual parameters. Other items will be investigated such as the impact of the number of individuals on the calibration process and how the results of the calibration depend on the involved specimens.

Throughout this work, the following indicators will be studied:

- The Kullback-Leibler divergence [58] between the exact $f_{\text {exact }}$ and the approximated distribution $\hat{f}_{\text {calibrated }}: \operatorname{KL}\left(f_{\text {exact }}, \hat{f}_{\text {calibrated }}\right)=\int_{\mathbb{R}^{d}} f_{\text {exact }}\left(\boldsymbol{\theta} \mid \Psi_{\text {exact }}\right) \ln \left(\frac{f_{\text {exact }}\left(\boldsymbol{\theta} \mid \boldsymbol{\Psi}_{\text {exact }}\right)}{\left.\hat{f}_{\text {calibrated }} \boldsymbol{\theta} \mid \hat{\mathbf{\Psi}}\right)}\right) \mathrm{d} \boldsymbol{\theta}$

- Error in the parameters space : for all the individual parameters set, it reads as $\frac{1}{n} \sum_{i=1}^{n} \frac{\left|\boldsymbol{\theta}_{\mathbf{i}, \text { exact }}-\hat{\boldsymbol{\theta}}_{\boldsymbol{i}}\right|}{\boldsymbol{\theta}_{\mathbf{i}, \text { exact }}}$ (division is elementwise, with no target value equal to 0 )

- Error of distribution parameters : $\frac{\left|\Psi_{\text {exact }}-\hat{\Psi}\right|}{\Psi_{\text {exact }} \mid}$ (division is elementwise, with no target value equal to 0 )

- Error in model space : for all the experiments, it can be written as $\frac{1}{n} \sum_{i=1}^{n} \frac{1}{N_{i}}\left\|\sigma_{i, \text { calc }}-\mathcal{F}\left(\mathbf{t}_{i}, \hat{\boldsymbol{\theta}}_{\boldsymbol{i}}\right)\right\|_{2}$

Only the last criterion can be used to study the results obtained with experimental data because in this case neither the exact model parameters distribution nor the exact individual parameters are known.

Several Assumptions are made here. First, only monotonic loadings are considered so that, from the original 6 parameters of the ODM model, the residual strain component can be neglected. Consequently, $\chi$ is set to 0 because this parameter is only involved in the computation of the residual strain component. In addition, the material model implies strong correlations such as those between $y_{c}^{s}$ and $p$ or $y_{0}^{s}$ and $p$. To avoid correlations at the beginning, it is decided to set $p$ to 1.2, a usual value for this kind of materials. As a consequence, all the parameters are considered as independent and the covariance $\Sigma$ is a diagonal matrix made of the variance terms. This simplifying assumption is discussed in the perspectives of the paper. It is also decided to assign to each specimen the same error term

$$
\forall(i, j) \in \llbracket 1, n \rrbracket \times \llbracket 1, N_{i} \rrbracket \omega_{i j}=\omega
$$


Table 2: Exact values of the parameters distribution.

\begin{tabular}{lrrrr}
\hline & $E_{0}[\mathrm{MPa}]$ & $y_{0 s}[\mathrm{MPa}]$ & $y_{c s}[\mathrm{MPa}]$ & $d_{c}$ \\
\hline Mean & $1.89 \times 10^{5}$ & $2.50 \times 10^{-2}$ & 2.24 & 3.77 \\
\hline Standard deviation & $1.79 \times 10^{4}$ & $8.00 \times 10^{-3}$ & 0.439 & 0.893 \\
\hline
\end{tabular}

As a result, 8 to 9 parameters have to be calibrated (depending on if $\omega$ is calibrated or fixed which stand for 4 mean and 4 variance parameters.

For the computation of the likelihood with the importance sampling scheme, $10^{4} \mathrm{MCMC}$ samples are generated and the first 500 samples burnt. The thinning is set to 20. Once all these samples are discarded, it remains 4750 of the $10^{4}$ initial samples. The MCMC sampler used can be found in [59]. The algorithm used to minimize the function $g_{i}(\cdot)$ (Eq.(23)) is the CMA-ES algorithm [60] with a budget of $10^{3}$ iterations and a population size of 50 .

The algorithm that maximizes the likelihood function is the EGO algorithm from the GPy library [61]. The initial DoE is a latin hypercube sampling with 45 points to which 160 points are added during the optimization. Variables are normalized between 0 and 1. Optimization bounds are discussed in the hereafter.

\subsection{Calibrating with virtual data}

\subsubsection{Generating virtual data}

The targeted mean and standard deviations of the parameters are chosen at values which are consistent with usual observations as presented in Table 2. 20 i.i.d samples from $f_{\Psi_{\text {exact }}}$ are generated. For each of these samples and given a strain profile (the strain rate $\dot{\varepsilon}=\frac{\partial \varepsilon}{\partial t}$ is equal to $\left.3.00 \times 10^{-4} \mathrm{~s}^{-1}\right)$, the corresponding model outputs $\left(\sigma_{i, \text { calc }}\right)_{i \in \llbracket 1,20 \rrbracket}$ are computed. An heteroscedastic noise is added to the experimental data:

$$
\sigma_{i, \text { noisy }}=\sigma_{i, \text { calc }} \times(1+\beta \zeta) \text { with } \zeta \sim \mathcal{N}(0,1) \text { and } \beta=0.02
$$

Exact and noisy data are depicted in Figure 2. The bounds on both means and standard deviations used for the optimization are shown in Tables 3 and 4. Within these bounds, the search space of the model parameter distribution is large and the different distributions exhibit significant variety. This can be seen in Figure 3 where some marginals for different means and variances of $E_{0}$ are represented.

\subsubsection{Calibration with a fixed error}

This section is dedicated to the presentation of the results of the calibration of the ODM model with the virtual data presented in Figure 2. The results from both approaches (Laplace and MCMC-IS) are presented. The standard deviation of the error $\omega$ is fixed to $1 \mathrm{MPa}$. The 10 specimens involved in the calibration process are presented in Figure 2. The presented calibration process is run with the different settings introduced in Section 3.2.

To analyze the results, it is possible to look at the calibrated parameters distribution characterized by its mean and standard deviation. These values are presented in Table 5. The first comment is that the calibrated distributions are rather close to the exact distributions, except 


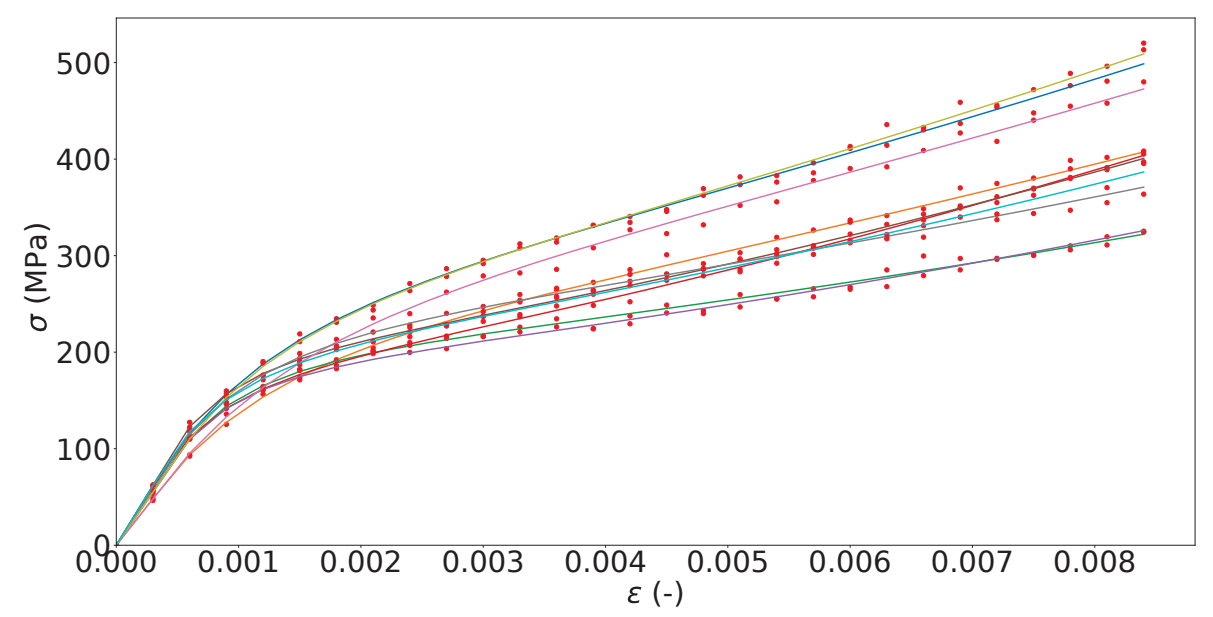

Figure 2: Model with exact individual parameters (lines) and noisy data (dots).

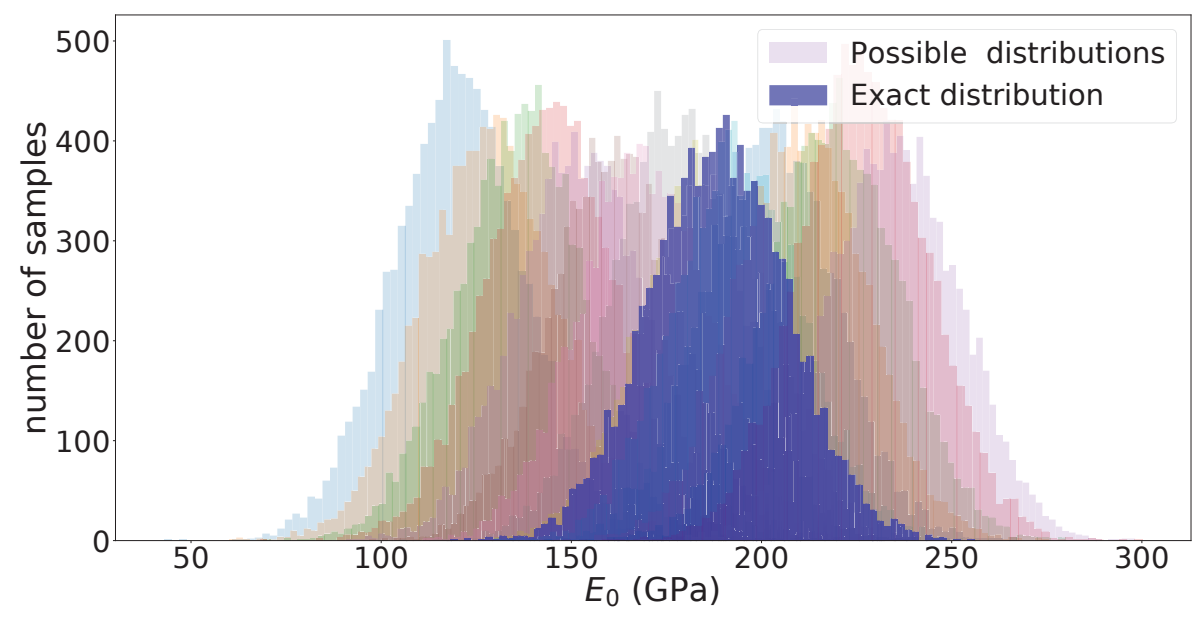

Figure 3: Possible marginal distributions for the parameter $E_{0}$.

Table 3: Bounds for the mean parameters $\boldsymbol{\mu}$.

\begin{tabular}{rrrrr}
\hline & $E_{0}[\mathrm{MPa}]$ & $y_{0 s}[\mathrm{MPa}]$ & $y_{c s}[\mathrm{MPa}]$ & $d_{c}$ \\
\hline Lower bound & $1.20 \times 10^{5}$ & $5.00 \times 10^{-3}$ & 1.10 & 1.30 \\
\hline Upper bound & $2.35 \times 10^{5}$ & $5.00 \times 10^{-2}$ & 3.50 & 6.00 \\
\hline
\end{tabular}

Table 4: Bounds for the standard deviation parameters, $\Sigma$.

\begin{tabular}{rrrrrr}
\hline & $\sigma_{E_{0}}[\mathrm{MPa}]$ & $\sigma_{y_{0 s}}[\mathrm{MPa}]$ & $\sigma_{y_{c s}}[\mathrm{MPa}]$ & $\sigma_{d_{c}}$ & $\omega[\mathrm{MPa}]$ \\
\hline Lower bound & $1.40 \times 10^{4}$ & $3.00 \times 10^{-3}$ & 0.250 & 0.600 & 0.381 \\
\hline Upper bound & $2.00 \times 10^{4}$ & $1.00 \times 10^{-2}$ & 0.500 & 1.08 & 12.5 \\
\hline
\end{tabular}


for $y_{0 s}$. The corresponding marginals are displayed in Figures 5a, 5c, 5d. Both approaches seem to provide a different result as as two order of magnitude separate their Kullback-Leibler divergences (see Table 7). However, omitting the $y_{0 s}$ makes the KL divergence fall to 0.81 for the MCMC approach and 0.33 for the Laplace approach. The two approaches provide similar results except for the damage threshold. The marginal distributions associated to the Young's modulus and the damage evolution celerity are well calibrated. The distribution associated to the Young's modulus is generally the best calibrated parameter distribution as it appears to be the most sensitive parameter in the ODM model, followed by $y_{c s}$ and $d_{c}$. The differences between the different coordinates are further discussed later.

On Figure 6, the resulting calibrated individual parameters value for each specimen is displayed. It is important to notice that the values of the individual model parameters are all different allowing to express the variability of the material specimen. This is a key aspect of the proposed approach compared to existing techniques discussed in the introduction. Considering the results about the individual model parameters, the mean relative errors (over the 10 individuals) between the exact and the calibrated individual parameters is presented in Table 8. The relative error on the Young's modulus, which is from both a numerical and mechanical points of view the most sensitive parameters, is the smallest with respect the other mean errors. The damage saturation $d_{c}$ and the damage evolution celerity $y_{c s}$ are also properly calibrated in both approaches. However, as it was possible to expect, the mean of relative errors on $y_{0 s}$ is the highest in each approach. It is also possible to check that the data used in the calibration process is properly calibrated. For that, the model outputs corresponding to the estimated parameters are plotted in Figure $7 \mathrm{a}$ in which it is possible to notice that the model is properly calibrated as the model responses are in adequation with the virtual data. This can be assessed by checking the averaged distance in the model space given in Table 9. For each specimen, the difference betwen the model responses with the exact individual parameters and with the calibrated individual parameters are illustrated in Figure $7 \mathrm{~b}$. It illustrates the efficient calibration of the ndividual model parameters for all the considered specimens. Even if the marginal distributions for the model parameters are assumed to be independent, in the MCMC-IS approach, the generated model parameter samples by the importance density using MCMC (Figure 8) exhibit the correlations between the parameter coordinates in order to generate model responses in adequation with the available virtual data.

Neither the marginals nor the individual parameters of the damage threshold $y_{0 s}$ are well calibrated. One of the hypotheses which can explain this fact is the lack of sensitivity of the damage threshold in the objective function. To check this assumption, it is possible to carry out a raw sensitivity analysis by studying the lengthscales of the Gaussian process involved in the optimization process. This interpretation comes from the fact that the lengthscale acts as a wavelength of the Gaussian process which is here a surrogate model of the mixed-effects likelihood function. If the wavelength along one axis of the Gaussian process is small, it means that the range of variations of the objective function along this direction is small, which may be interpreted as a limited sensitivity of the objective function along this coordinate. On the contrary, if the wavelength is large, the variations of the objective function along this direction exhibit large variations, which means the objective function varies significantly along this axis and the corresponding parameter is sensitive. For the Laplace calibration, the order of magnitude of the lengthscales (standard and normalized with respect to the optimization bounds) are presented in Table 6. Standard numerical values give $\ell\left(E_{0}\right) \gg \ell\left(d_{c}\right), \ell\left(y_{c s}\right)>\ell\left(y_{0 s}\right)$ as expected. Normalized lengthscales only indicate that $\ell\left(E_{0}\right) \gg \ell\left(d_{c}\right), \ell\left(y_{c s}\right), \ell\left(y_{0 s}\right)$, which is still consistent with physical knowledge. The low sensitivity of $y_{0 s}$ in the likelihood function, 


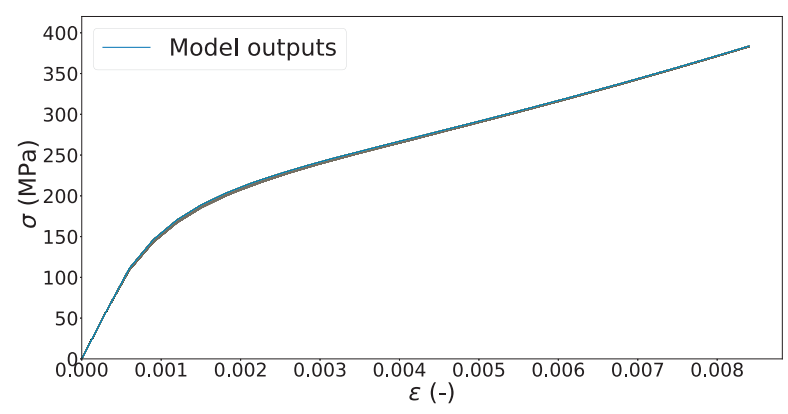

(a)

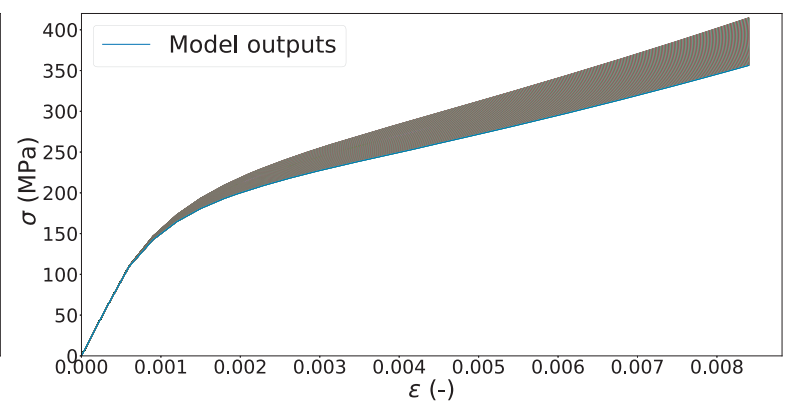

(b)

Figure 4: On the left (a), model outputs for different damage thresholds varying between $[0.9 \times$ $\left.\mu_{y_{0 s}}, 1.1 \times \mu_{y_{0 s}}\right]\left(\mu_{y_{0 s}}\right.$ and other parameters correspond to the mean parameters presented in Table 1). On the right (b), model outputs for different damage thresholds varying between $\left[0.9 \times \mu_{y_{c s}}, 1.1 \times \mu_{y_{c s}}\right]\left(\mu_{y_{c s}}\right.$ and other parameters correspond to the mean parameters presented in Table 1).

which explains the differences encountered along the $y_{0 s}$ (Figure 5b) axis can come from the choice of $p$. In Section 3.1, it was stated that $y_{0 s}$ was complicated to calibrate for $p>1$ with only strain-stress curves, which means that previous remarks are consistent with expert knowledge. To investigate the sensitivity of the model ouput towards the damage threshold, another simple solution can consist in computing the model outputs for different damage threshold and the other parameters fixed. On Figure $4 \mathrm{a}$, it is possible to notice that the damage threshold, for a variation of $20 \%$ around the corresponding mean value $\mu_{y_{0 s}}$, does not influence much the model output as for all strain values, the difference between the extreme stress curves is beneath $5 \mathrm{MPa}$. The relative variation of the stress is below $5 \%$ which can be considered as non significant. Yet, the model responses exhibit greater sensitivity for the same relative variation of the damage evolution celerity $y_{c s}$ (see Figure $4 \mathrm{~b}$ ). The same observations can be made on the other model parameters. This ascertains the results obtained with the analyses on the lengthscales. In practice, $y_{0 s}$ is usually calibrated using acoustic data which are not available here. Damage starts when the first fibers of the composite material break. It produces acoustic events which can be recorded and help to indicate when the first failure (and the start of damage) happens.

In this section, the ability of the mixed-effects models methodology to calibrate the ODM model has been illustrated. This framework allows to characterize the material variability and its impact on the model parameters. In addition, to provide information on the distribution of the model parameters, it allows to determine reliable estimates of the individual model parameters. Furthermore, both computational methods of the likelihood (i.e. MCMC-IS and Laplace) give similar results in terms of calibrated model parameter distributions and individual parameter values. Given that the computation of the likelihood with the Laplace method is much faster to compute (for 10 specimens of 29 measurements each on a multi-threaded code with 4 CPU on a standard machine with processor Intel Core I5, MCMC-IS approach takes 2 minutes to compute the likelihood while the Laplace approach takes about 4 seconds), all further analyses will be conducted using the Laplace approximation method for the computation of the likelihood. 


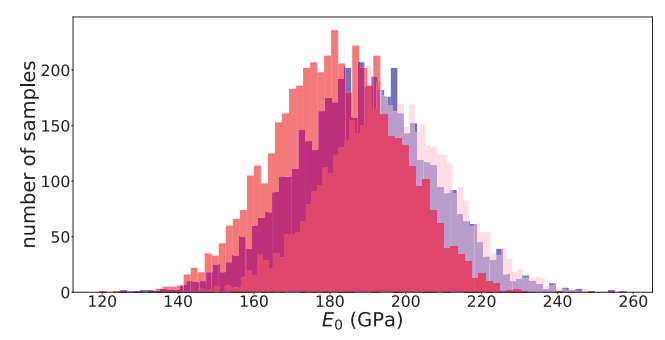

(a)

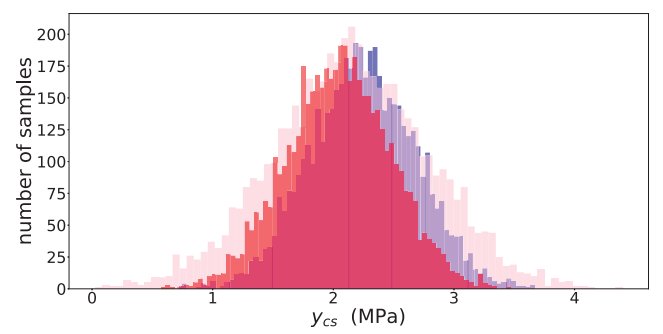

(c)

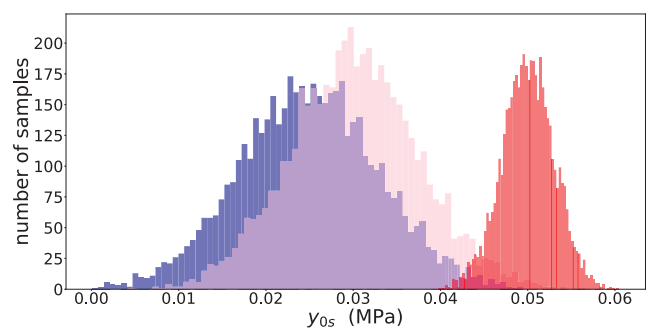

(b)

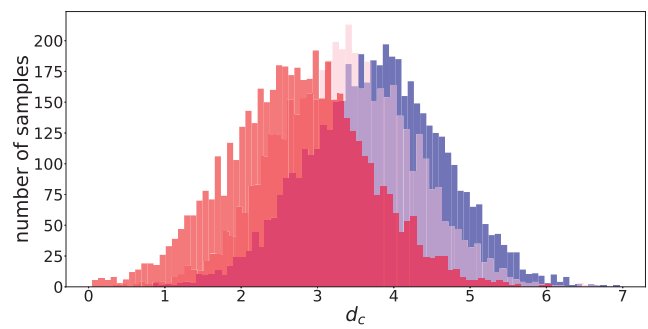

(d)

Exact distribution

MCMC calibrated distribution with 10 individuals

Laplace calibrated distribution with 10 individuals

Figure 5: Exact and calibrated marginals along the $E_{0}$ axis (a), the $y_{0 s}$ axis (b), the $y_{c s}$ axis (c), and the $d_{c}$ axis $(\mathrm{d})$.

Table 5: Calibrated means and standard deviations with fixed error term. The relative error between the exact $\Psi$ and the calibrated $\Psi$ in $\%$ is indicated between brackets.

\begin{tabular}{lrrrr}
\hline & $E_{0}[\mathrm{MPa}]$ & $y_{0 s}[\mathrm{MPa}]$ & $y_{c s}[\mathrm{MPa}]$ & $d_{c}$ \\
\hline Exact means & $1.88 \times 10^{5}$ & 0.0250 & 2.24 & 3.77 \\
\hline $\begin{array}{l}\text { Laplace cali- } \\
\text { brated means }\end{array}$ & $1.95 \times 10^{5}(4)$ & $0.0301(20)$ & $2.15(4)$ & $3.36(10)$ \\
\hline $\begin{array}{l}\text { MCMC-IS cali- } \\
\text { brated means }\end{array}$ & $1.81 \times 10^{5}(4)$ & $0.0500(100)$ & $2.05(8)$ & $2.74(27)$ \\
\hline Exact standard deviations & $1.79 \times 10^{4}$ & $8.00 \times 10^{-3}$ & 0,439 & 0,893 \\
\hline $\begin{array}{l}\text { Laplace calibrated } \\
\text { standard deviations }\end{array}$ & $1.62 \times 10^{4}(9)$ & $7.31 \times 10^{-3}(9)$ & $0.647(47)$ & $0.871(2)$ \\
\hline $\begin{array}{l}\text { MCMC-IS calibrated } \\
\text { standard } \\
\text { deviations }\end{array}$ & $1.67 \times 10^{4}(7)$ & $3.00 \times 10^{-3}(62)$ & $0.431(1)$ & $0.910(2)$ \\
\hline
\end{tabular}




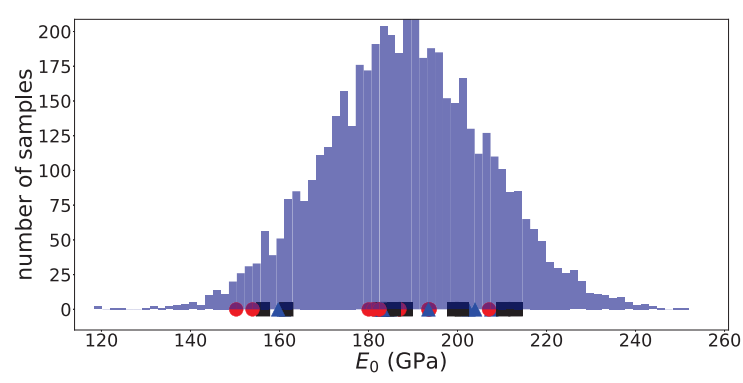

(a)

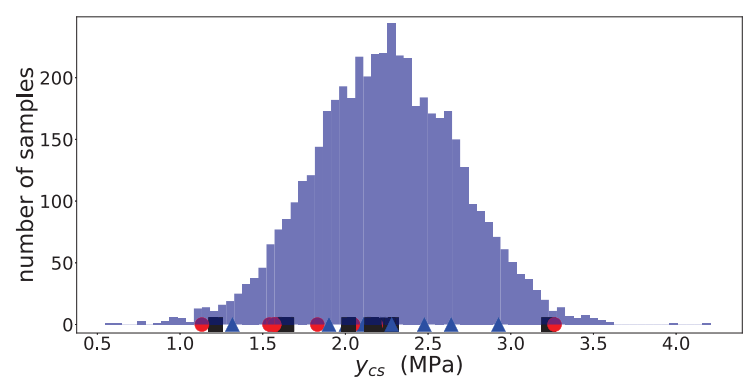

(c)

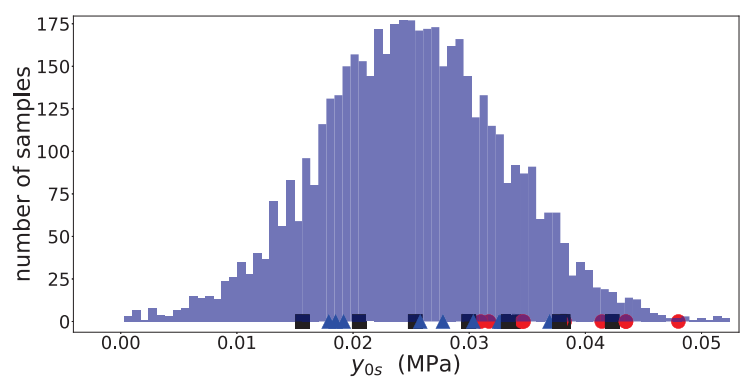

(b)

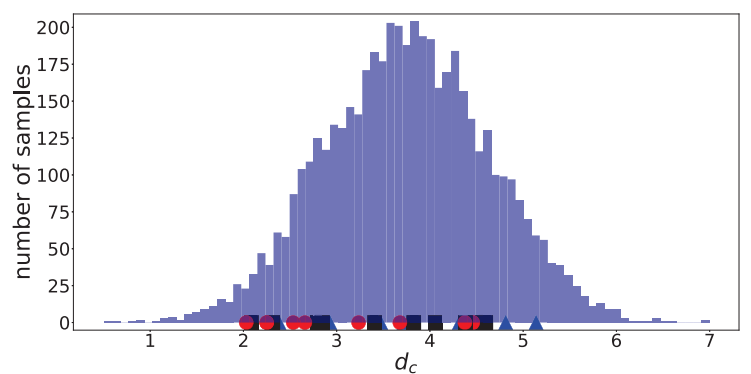

(d)

\footnotetext{
Exact distribution

- Exact individual parameters

- Laplace individual parameters

- MCMC individual parameters
}

Figure 6: Exact marginals, exact and calibrated individual parameters along the $E_{0}$ axis (a), the $y_{0 s}$ axis (b), the $y_{c s}$ axis (c), and the $d_{c}$ axis (d).

Table 6: Order of magnitude of the Gaussian process lengthscale along each direction at the the last iteration for the Laplace calibration process.

\begin{tabular}{lrrrr}
\hline & $\ell\left(E_{0}\right)$ & $\ell\left(y_{0 s}\right)$ & $\ell\left(y_{c s}\right)$ & $\ell\left(d_{c}\right)$ \\
\hline standard lengthscale $\ell$ & $10^{9}$ & $10^{-2}$ & $10^{0}$ & $10^{0}$ \\
\hline normalized lengthscale $\ell_{r}$ & $10^{4}$ & $10^{-1}$ & $10^{-1}$ & $10^{-1}$ \\
\hline
\end{tabular}

Table 7: Kullback-Leibler divergences for the virtual data case, the error term being fixed.

\begin{tabular}{rrr}
\hline & MCMC-IS & Laplace \\
\hline Kullback-Leibler divergence & 37.3 & 0,53 \\
\hline
\end{tabular}

Table 8: Mean relative errors for the 10 calibrated individual parameters compared to the exact parameter values.

\begin{tabular}{lrrrr}
\hline & $E_{0}[\mathrm{MPa}]$ & $y_{0 s}[\mathrm{MPa}]$ & $y_{c s}[\mathrm{MPa}]$ & $d_{c}$ \\
\hline Laplace relative error $(\%)$ & 1.59 & 23.5 & 10.6 & 3.25 \\
\hline MCMC-IS relative error $(\%)$ & 3.04 & 43.15 & 13.4 & 6.04 \\
\hline
\end{tabular}


Table 9: Mean approximation error for the 10 model outputs corresponding to the calibrated individual parameters compared to model outputs corresponding the exact parameter values.

\begin{tabular}{rrr}
\hline & Laplace & MCMC-IS \\
\hline Distance in model space (MPa) & 0,311 & 0,348 \\
\hline
\end{tabular}

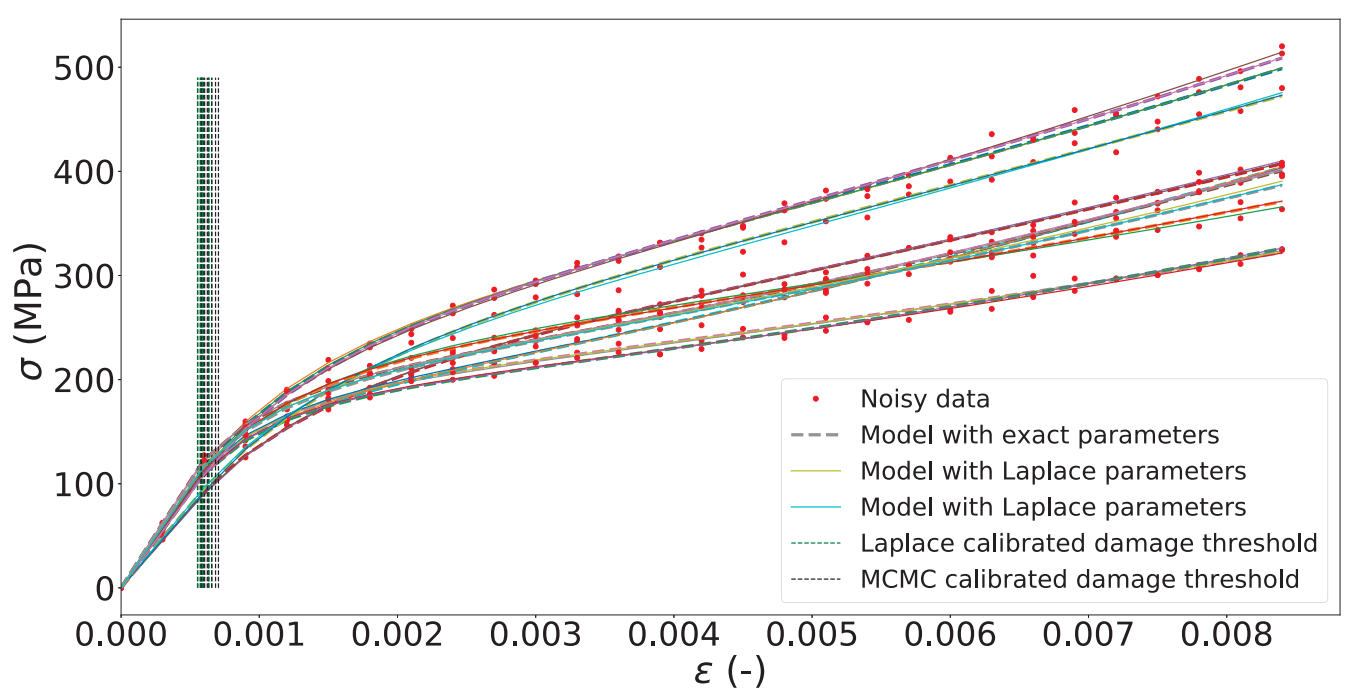

(a)

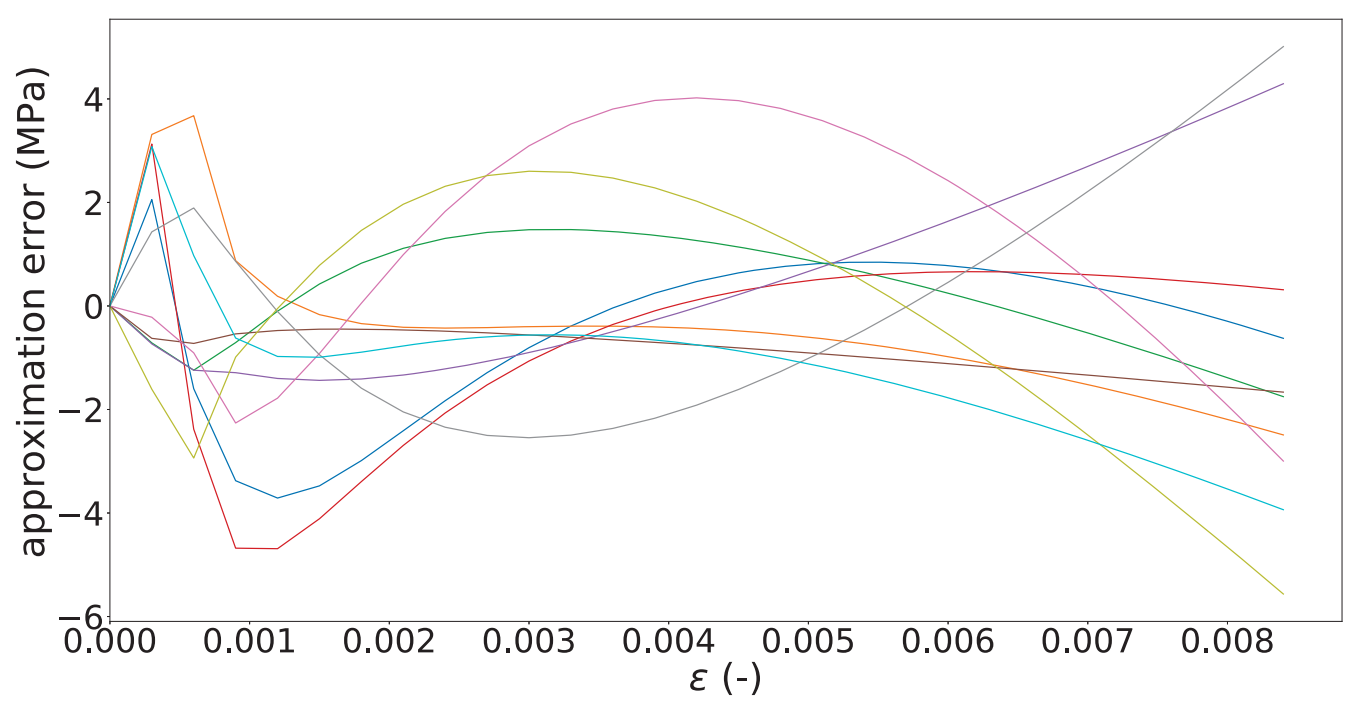

(b)

Figure 7: In (a), exact data used for calibration vs model responses for the estimated individual parameters. In (b), differences between model with exact parameters and with Laplace calibrated parameters. 

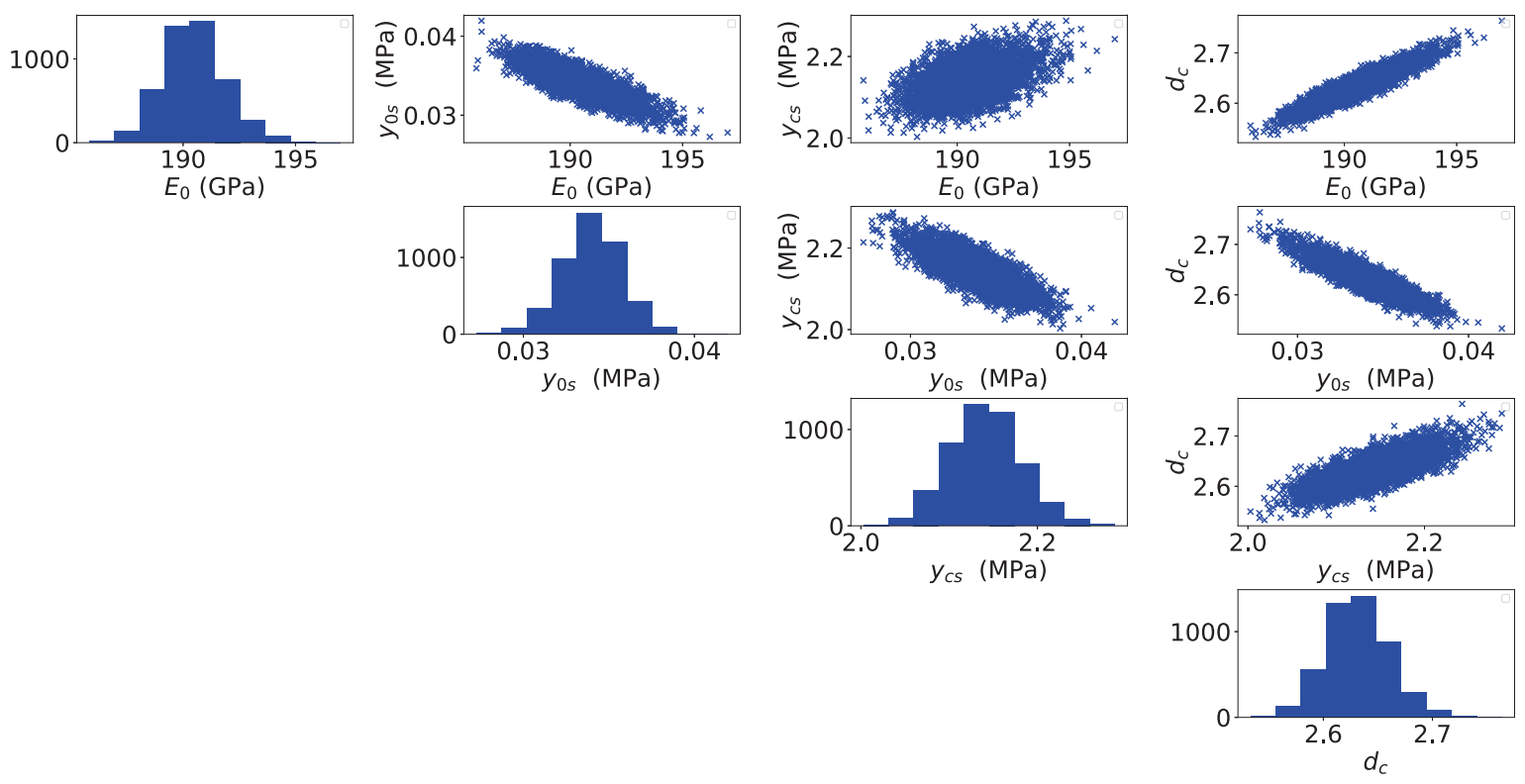

Figure 8: Pairplot of the MCMC samples of $\hat{f}\left(\boldsymbol{\theta} \mid \mathbf{y}_{0}, \hat{\Psi}\right)$.

\subsubsection{Calibration with an optimized error term}

In this Section, the variance $\omega$ of the error term is not considered fixed anymore and it is calibrated along with the other parameters in $\Psi$. Moreover, the objective of this section is to study the influence of the number of available individuals in the calibration method.

The number of available observations plays a key role in calibration. In general, a high number of observations allows to reduce the uncertainty associated to the calibrated parameters. However, in mechanics, the number of specimens is limited due to time and cost considerations and the usual number of specimens varies from 5 to 10 in the best cases. As a result, to study the influence of the number of individuals on the calibration results, it is decided to calibrate the model with respectively 5, 10 and 20 individuals (all depicted in Figure 2) to take into account representative constraints on the number of available specimens. The upper bound on the variance of the error term is set to $10 \mathrm{MPa}$ and the lower bound to $0.1 \mathrm{MPa}$.

The presented calibration approach using the Laplace technique for the likelihood estimation is carried out for 5, 10 and 20 individuals generated with the same exact model parameters distribution. To analyze the obtained results, it is possible to plot the marginals along the $E_{0}$ axis for 5, 10 and 20 individuals (see Figure 9). In this figure, it is possible to notice the marginal along the $y_{c s}$ axis is better inferred as the number of specimens increases. This phenomenon can also be noticed for the marginal along the $d_{c}$ axis (see Figure 17). However, the marginal along the $E_{0}$ axis is downgraded from 10 to 20 individuals (see Figure 16). As the marginal distributions are jointly calibrated, it is necessary to measure the improvement of the calibration using Kullback-Leibler divergences for 5, 10 and 20 individuals ( $y_{0 s}$ is ommited in the KL calculation, see discussion in the previous section). The KL divergence decreases with the increase of the number of individuals (Table 10), therefore the distribution of the model parameters is better calibrated as the number of available specimens increases. In addition, on Figure 11b, it can be noticed that the damage threshold is always the parameter with the highest associated error: in other words, this parameter is complicated to calibrate regardless of the number 
Table 10: Kullback-Leibler divergences for 5, 10 and 20 individuals (without consideration of $y_{0 s}$ marginal).

\begin{tabular}{lrrr}
\hline Number of specimens & 5 & 10 & 20 \\
\hline Kullback-Leibler divergence & 0.82 & 0.42 & 0.32 \\
\hline
\end{tabular}

Table 11: Mean error (for all the deformation values) for 5, 10 and 20 individuals between the calibrated model responses and the model responses with the exact parameters.

\begin{tabular}{lrrr}
\hline Number of specimens & 5 & 10 & 20 \\
\hline Mean approximation error (MPa) & 0.226 & 0.327 & 0.357 \\
\hline
\end{tabular}

of specimens. To illustrate the impact of the model parameter distribution convergence on the model responses, it is possible for each calibrated distribution (for 5, 10 and 20 individuals) and for the exact distribution to generate parameter samples (2000 in the present case). Then, for each parameter sample, the ODM model is computed. Eventually, the $5 \%$ and $95 \%$ quantiles in the model response space are estimated (Figure 10).

From Figure 10, it can be seen that the estimated $5 \%$ quantiles for the model responses using the calibrated distribution and the exact distribution converge one to another as the number of individuals increases. Similar analysis can be done for the $95 \%$ quantiles. It allows to see that the convergence of the calibrated model parameters distribution toward the exact distribution as the number of individuals increases, enables the generation of model responses that cover the entire set of possibilites (and not just a subset as for 5 individuals). This is of prime importance if this calibrated model parameter distribution is then used for uncertainty propagation in structure analysis for instance. It is therefore important to keep in mind that with a small number of individuals (around 5 specimens), the calibrated model parameter distribution might not be enough representative of the entire set of possible outcomes (due to the lack of individual diversity with only 5 specimens). Therefore, with a limited number of specimens, the calibrated model parameter distribution has to be used with attention.

Eventually, it is important to notice that the resulting model parameter values for each individual is closed to the exact parameter values even when only 5 individuals are considered.

In this section, the sensitivity to the number of individuals has been studied. It was has been that as the number of specimens increases, the calibrated marginals get closer to the exact marginals, and the same thing is observed in the model space. Finally, it was also noticed even with few specimens, the mixed-effects methods calibrates well the involved specimens.

\subsubsection{Robustness of the model calibration with respect to different available individuals}

Calibration in the mixed-effects models framework relies on specimens to determine the model parameter distribution and later estimate the individual parameters values. The objective of this section is to evaluate the robustness of the calibration using mixed-effects models with respect to the available specimens. In other words, whatever the specimens are, the population- 


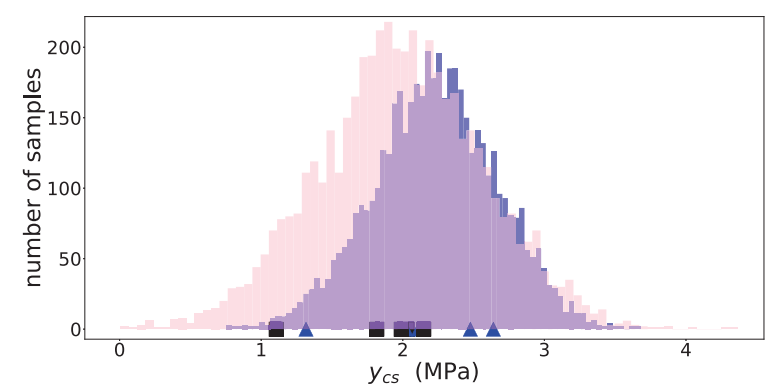

(a)

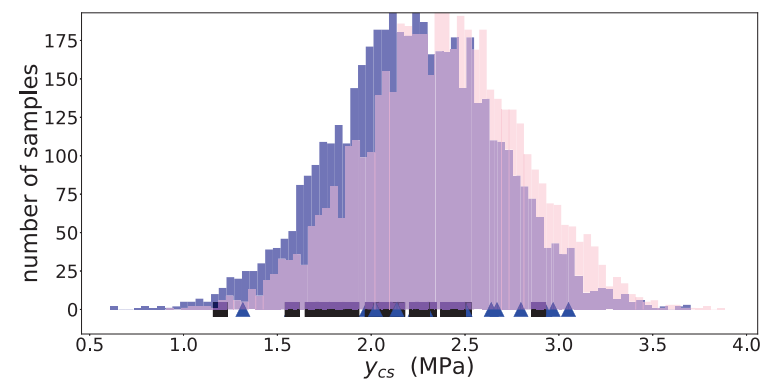

(c)

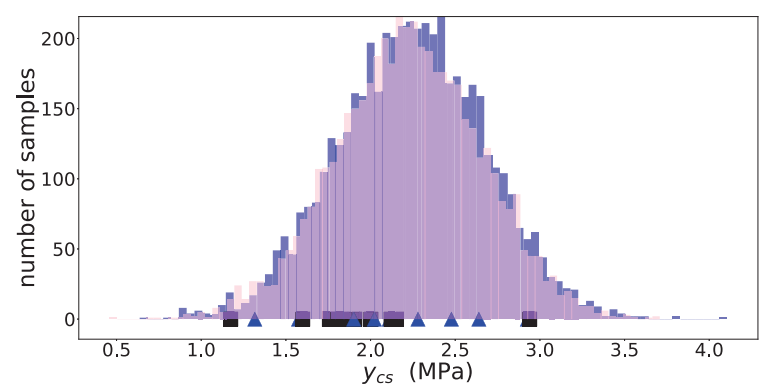

(b)

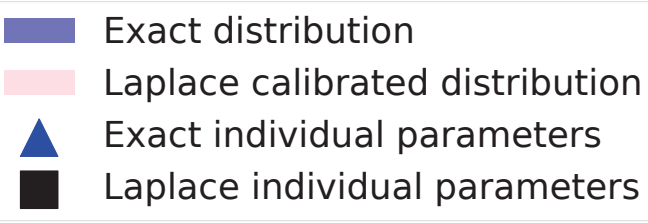

Figure 9: Exact and calibrated marginals along the $y_{c s}$ axis for 5 individuals (a), 10 individuals (b) and 20 individuals (c).

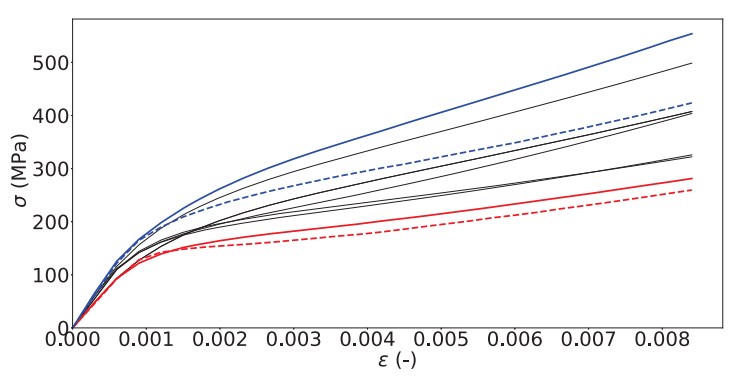

(a)

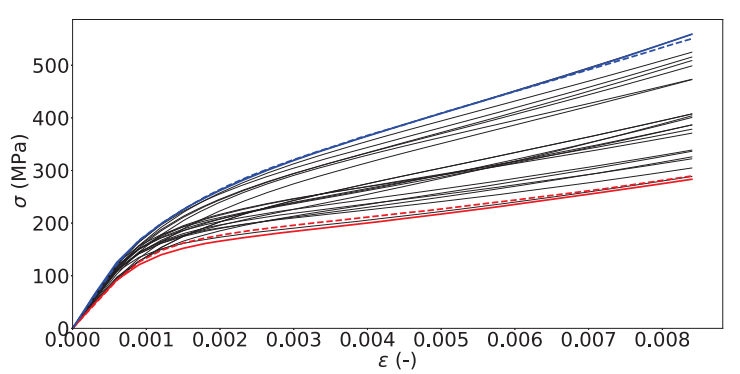

(c)

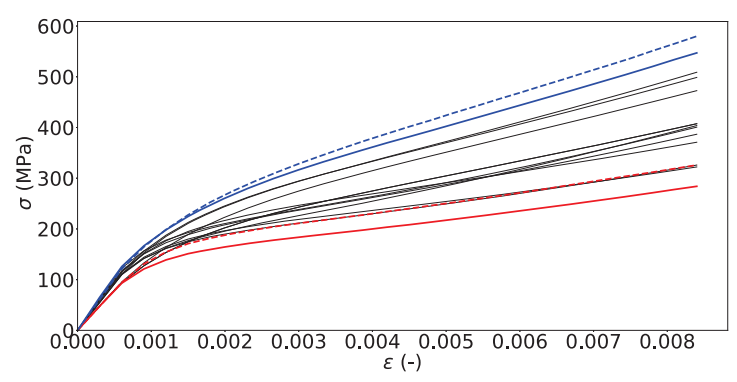

(b)

- Model with exact parameters

- $5 \%$ quantile for the exact model distribution

- $95 \%$ quantile for the exact model distribution

- - - $5 \%$ quantile for the calibrated model distribution

- - - $95 \%$ quantile for the calibrated model distribution

Figure 10: Uncertainty propagation from the distribution calibrated with 5 individuals (a), 10 individuals (b) and 20 individuals (c). 
based approach is able to approximate correctly the model parameter distribution and the individual parameters values. To check this assumption, the calibration process is repeated 20 times with different sets of specimens. For each of these repetitions, 10 different individual parameters values set are generated from the same exact distribution $f_{\Psi_{\text {exact }}}$, the corresponding model outputs are computed and noise is added as in Eq.(33). The individual parameters are different from one repetition to the other. All the repetitions are calibrated with mixed-effects models methodology using the same settings (i.e. initial DoE, number of iterations, bounds, etc.).

For each repetition, the Kullback-Leibler divergence is computed (see Figure 11a). The advantage of the Kullback-Leibler divergence is that this quantity takes into account all the different variations of the joint distribution. It is also possible to compute the mean error between the estimated individual parameters and the exact individual parameters for the 10 considered individuals and that for the 20 repetitions (see the boxplots 11b).

The objective is to check that calibration using mixed-effects models approach does not depend of involved specimens. With Figure 11a and Figure 11b, it is possible to notice that for all the repetitions, the range of variation of the Kullback-Leibler divergences and the mean relative errors are rather small. This tends to indicate that for the distribution parameters, the calibration results do not depend too much to the involved specimens. It is also possible to consider the calibrated variances of the error term. The different calibrated standard deviations of the error terms with 10 specimens are shown in Figure 11c. For all the repetitions but one, the standard deviation of the error term varies between 4.53 to $6.41 \mathrm{MPa}$. As the hypothesis made on the noise in the likelihood function is different from the noise which was added to the virtual data, there is not any reference value. Nevertheless, it can be noticed that calibrated standard deviations are within a rather small range, which tends to confirm that the calibration of this parameter is rather independent of the specimens used for calibration.

Up to now, virtual data have been used in the calibration process in order to illustrate the approach with a pedagogical point of view. It allowed to compared to obtained calibration results with respect to a known model parameters distribution and to the known individual parameters values. With this virtual data, the sensitivity to the number of individuals and their repartitions have been analyzed.

In the next section, a test case using experimental data is carried out, using the same calibration process.

\subsection{ODM model calibration with experimental data}

We now apply the mixed-effects models to calibrate the ODM model with 13 tensile tests performed on CERASEP A400, a woven composite material. The available tests are plotted in Figure 12. The methology settings (i.e. initial DoE, number of iterations, kernel of the Gaussian process) are the same as in the previous sections. The calibration bounds are given in Tables 12 and 13.

Notice in Figure 12 that all curves are less disparate than the virtual data generated in Section 3.3.1. Material failure happens at different strains and thus the ultimate tensile strength varies. Moreover, the transition between the linear and non-linear regimes is also subjected to variability. These obervations constitute an interesting test case for a population-based approach such as the mixed-effects models. Finally, the experiments have different number of observations 


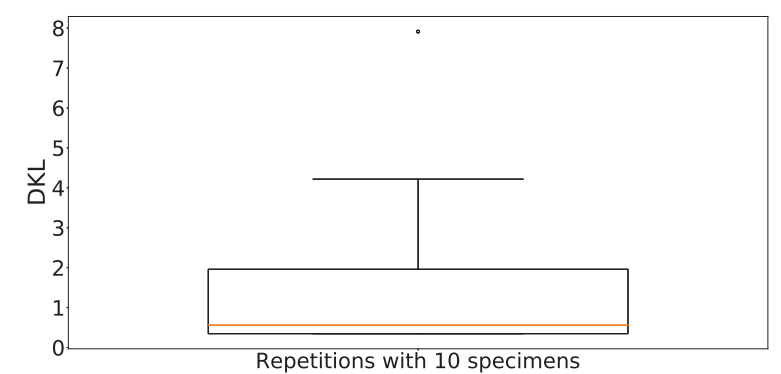

(a)

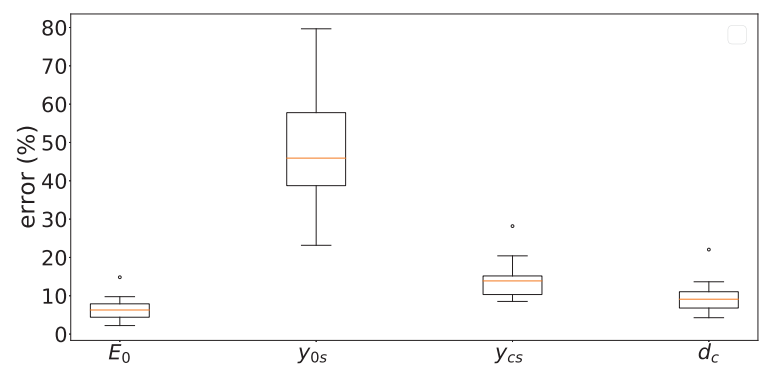

(b)

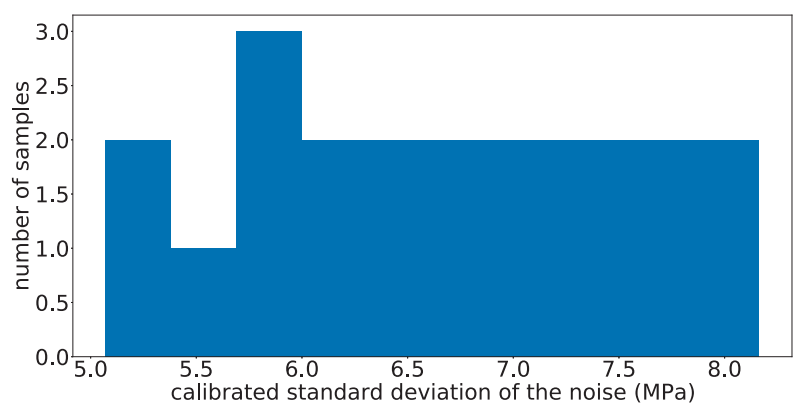

(c)

Figure 11: With 10 specimens: (a) boxplot of Kullback-Leibler divergence for all repetitions, (b) boxplot of the error of the individual parameters for all repetitions, (c) histogram of the calibrated standard deviation of the error term.

points (from 20 to 700). To make sure all individuals have the same weight in the likelihood function, it is decided to subsample those with more than 20 observation points. Experiments are subsampled peridiodically and always include the last point.

Figure 13 shows the calibrated marginals. The marginal along $E_{0}$ seems well calibrated in the sense that all the individual parameters belong to the interval $\left[\mu_{E_{0}}-3 \sigma_{E_{0}}, \mu_{E_{0}}+3 \sigma_{E_{0}}\right]$ which concentrates $99 \%$ of possible values for a Gaussian random variable. Along the $y_{0 s}$ axis, the same situation as as in Section 3.3 is encountered as all the individual parameters are concentrated around the mean. The individual parameters are also concentrated around the mean for $y_{c s}$ so that the variance seems overestimated. An explanation could be that the Bayesian optimization has not converged yet to the maximum likelihood estimator of $\Psi$. Along the $d_{c}$ axis, the individual parameters seem consistent with the marginal even if without the point whose damage saturation is between 3 and 3.5, the variance of $d_{c}$ would have been considered as overestimated. More classically, the adequation between the model responses for the estimated individual parameters and the experimental data is displayed in Figure 14 for two individuals (others can be found in appendix, Figures 19 and 20). In both plots, the estimated individual properly matches the experimental data (the same applies to the other experiments, see Figures 19 and 20)).

Eventually, it is possible to propagate the uncertainties of the calibrated distribution to the model parameters and to compare the results to the experimental data. In Figure 15, 1000 samples from the calibrated distribution are drawn and the corresponding model outputs computed. Notice that the experimental data are included in the bundle of model outputs, which shows 


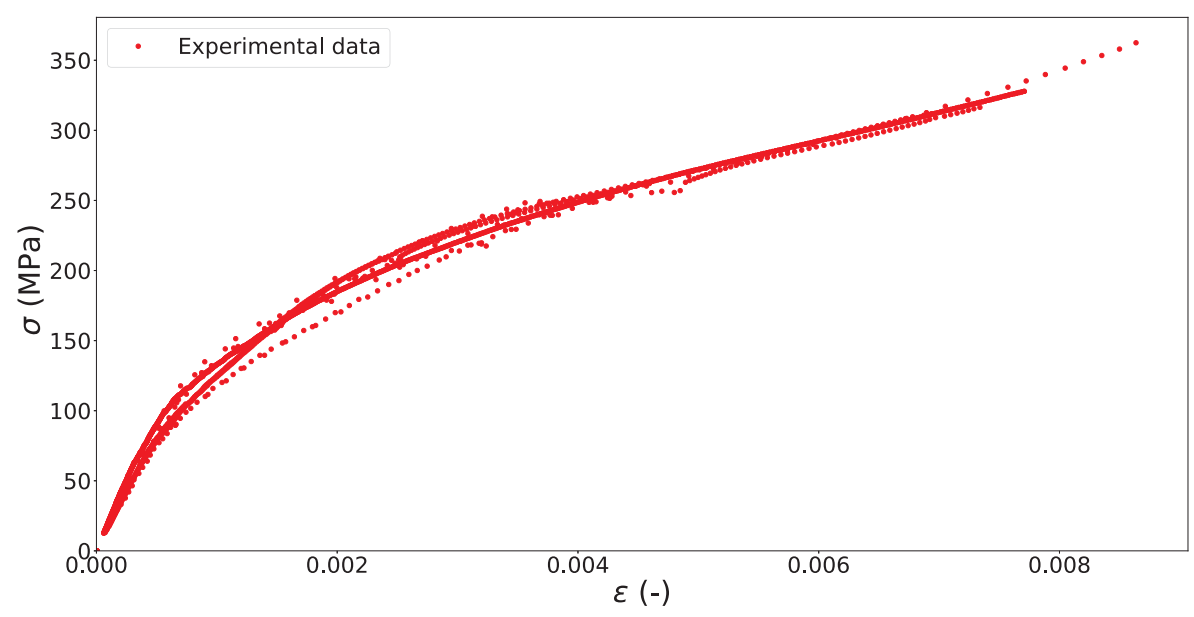

Figure 12: Experimental strain-stress curves of the CERASEP A400 material.

that the calibrated distribution conservatively learns the observed variability. The variability of the inferred distribution is more important than the one observed on the experimental curves. This overestimated variance of model parameters may be caused by the fact that the Bayesian optimzation has not converged yet with in the given number of iterations. Taking into account correlations between model parameters in the calibration process might help to reduce the estimated variances.

\section{CONCLUSIONS \& PERSPECTIVES}

In this paper, a population-based mixed-effects model was applied to calibrate a material model and to characterize material variability. First, the mixed-effects model has been described with an emphasis put on the numerical estimation of the likelihood. Then, this methodology has been applied to virtual data to compare the results with known model parameters distribution and individual parameters values. It has allowed to analyze the effects of the number of specimens and the specimens variability. Finally, the mixed-effects approach has been applied to experimental data obtained with a woven ceramic matrix composite material.

Our investigation show that mixed-effects models offer a promising framework to calibrate material parameters while taking into account specimen variability. Even if the accuracy of the calibrated parameter distribution seems sensitive to the number of specimens, the method typically provides accurate estimates of the individual parameters and captures well the observed variability. The number of specimens should also help to choose the level of details of the probabilistic model. For instance, calibrating correlations with a limited set of individuals yields estimates which may not be trustworthy.

In terms of future work, two major directions should be investigated. Firstly, it is important to include in the calibration process the correlations between the model parameters. A study of the relationship between the number of available individuals and the accuracy of the calibration correlations is essential. Secondly, improvements in the mixed-effects models are required in order to account for different types of data (for instance acoustic measures in addition to tensilestress observations). In doing so, the likelihood will be more sensitive to this parameter and as a matter, the marginal along the $y_{0 s}$ will be better calibrated and should make the estimation of the damage threshold more accurate. 


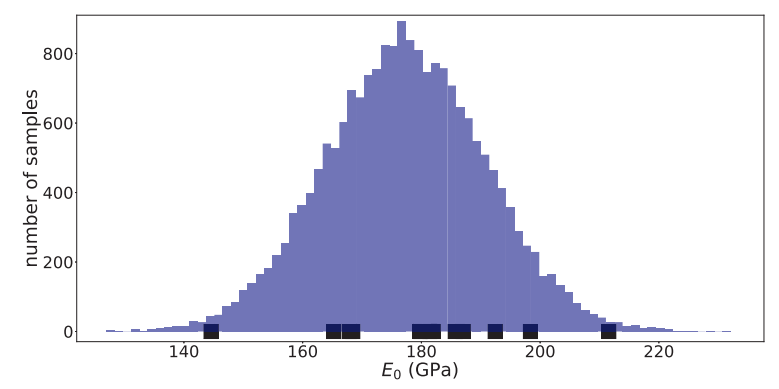

(a)

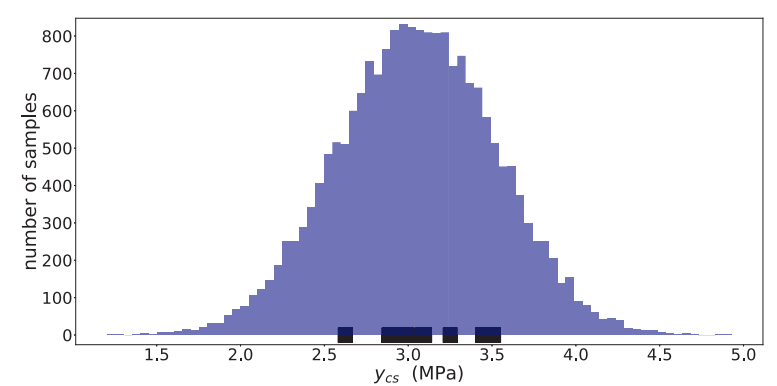

(c)

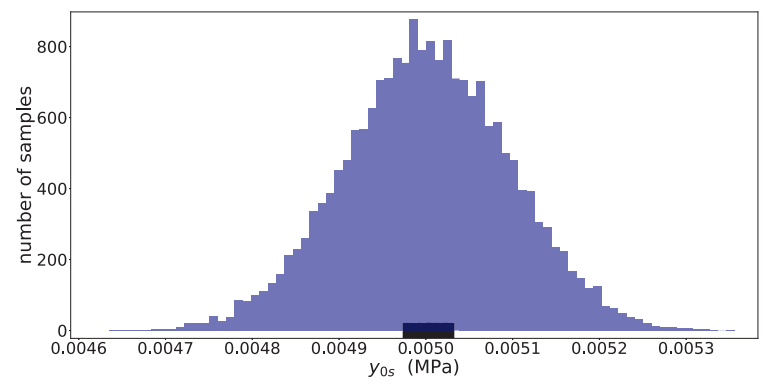

(b)

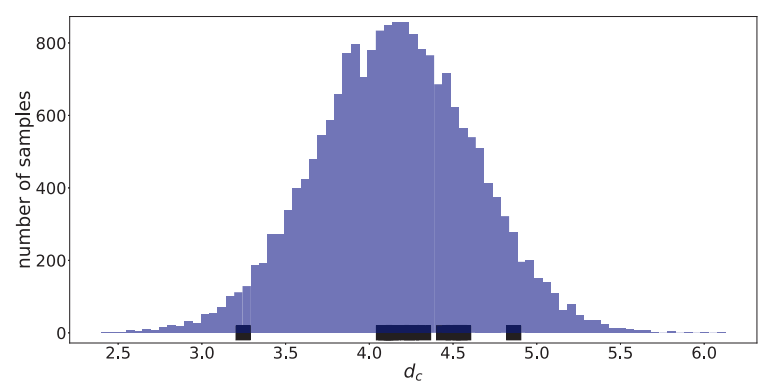

(d)

Laplace calibrated distribution with 13 individuals Laplace individual parameters

Figure 13: Calibrated model (histograms) and individual (squares) parameters along the $E_{0}$ (a), the $y_{0 s}(\mathrm{~b})$, the $y_{c s}(\mathrm{c})$ and the $d_{c}$ axes (d) using the 13 CERASEP A400 specimens.

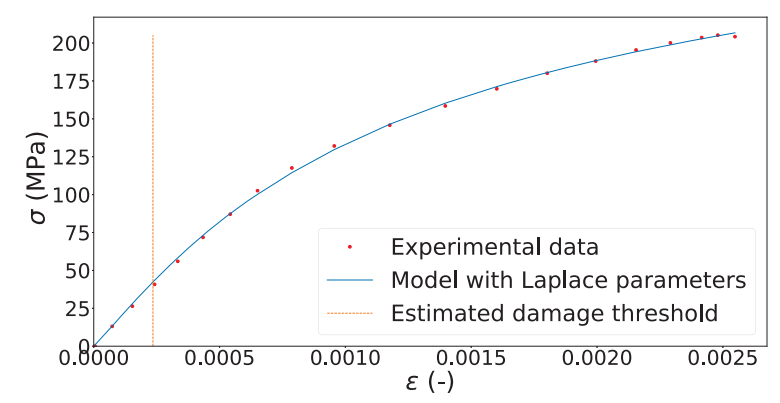

(a)

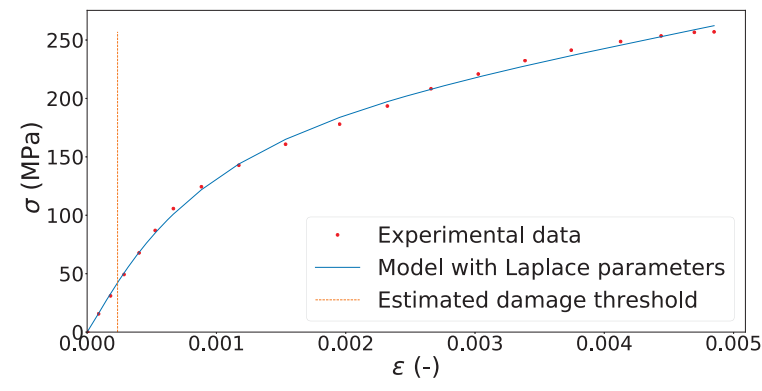

(b)

Figure 14: Model outputs vs. experimental data for the second (a) and the third (b) experiments. 


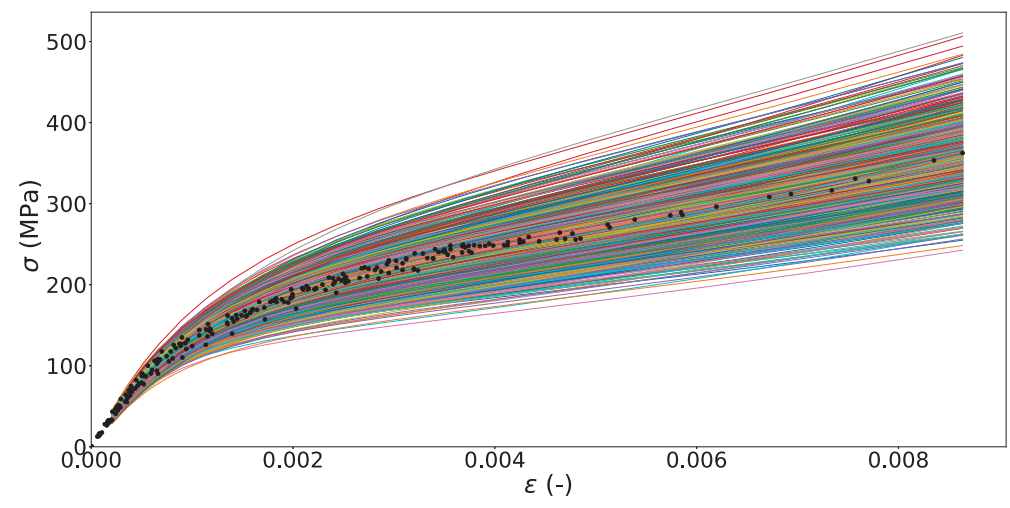

Figure 15: Uncertainty propagation from the calibrated distribution. 
A Calibrated model parameter marginals of $y_{c s}$ and $d_{c}$ with the Laplace approach using virtual data

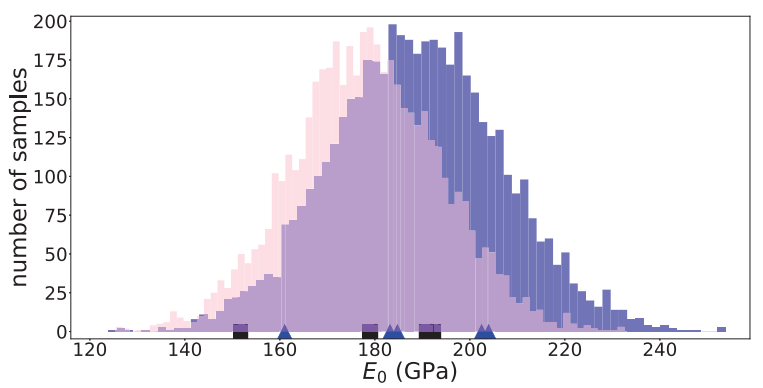

(a)

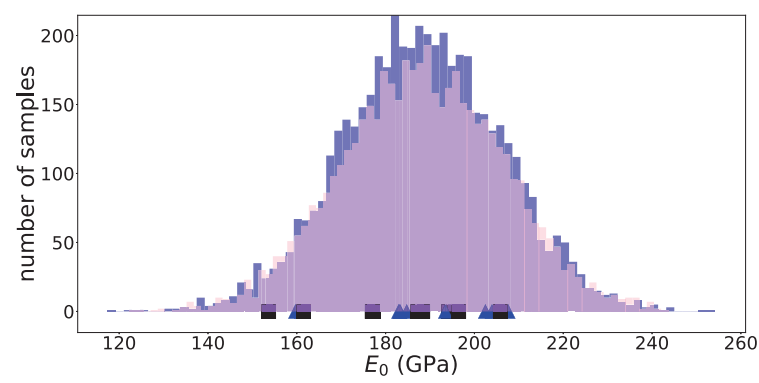

(b)

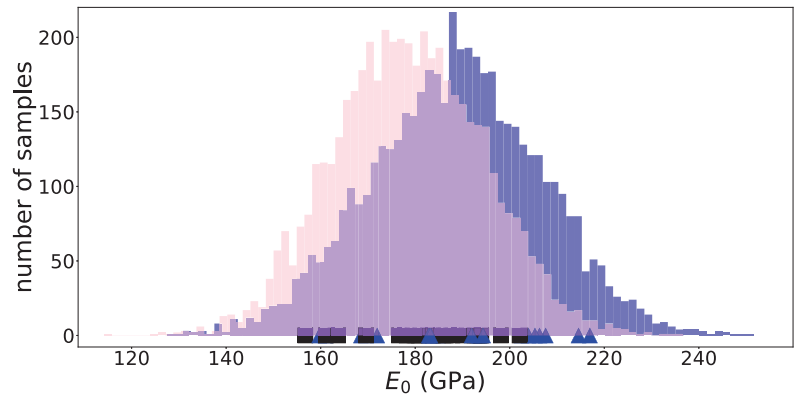

(c)

Figure 16: Exact and calibrated marginals along the $E_{0}$ axis for 5 individuals (a), 10 individuals (b) and 20 individuals (c). 
B Calibrated model parameter marginals of $d_{c}$ with the Laplace approach using virtual data

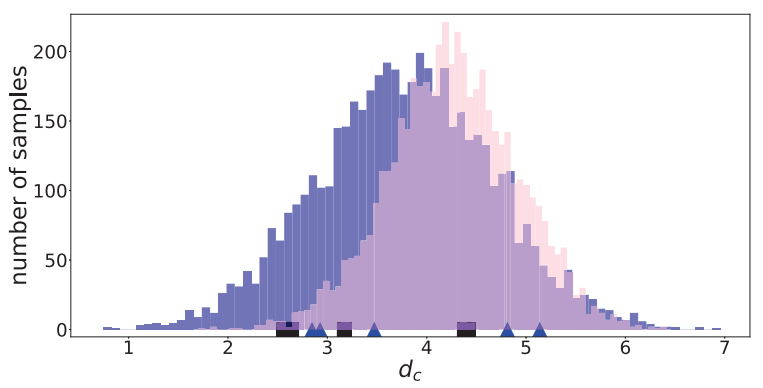

(a)

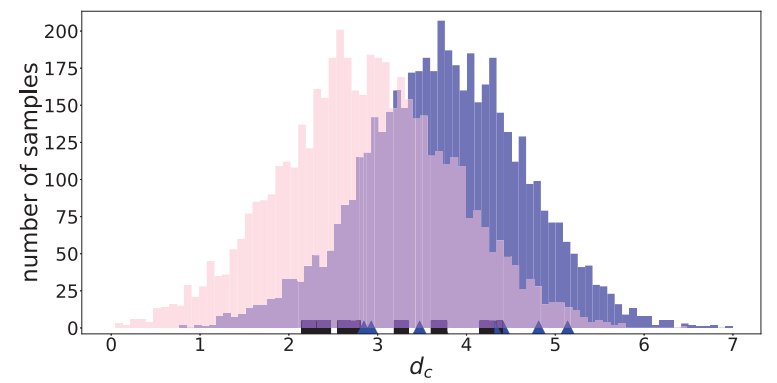

(b)

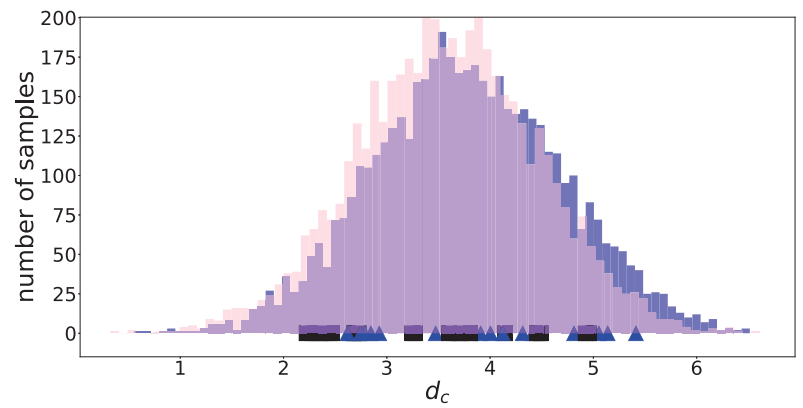

(c)

Figure 17: Exact and calibrated marginals along the $d_{c}$ axis with 5 individuals (a), 10 individuals (b) and 20 individuals (c). 


\section{Bounds of optimization for the Laplace approach}

Table 12: Bounds for mean parameters.

\begin{tabular}{rrrrr}
\hline & $E_{0}[\mathrm{MPa}]$ & $y_{0 s}[\mathrm{MPa}]$ & $y_{c s}[\mathrm{MPa}]$ & $d_{c}$ \\
\hline Lower bound & $1.40 \times 10^{5}$ & $5.00 \times 10^{-3}$ & 2.8 & 2.75 \\
\hline Upper bound & $2.2 \times 10^{5}$ & $5.00 \times 10^{-2}$ & 5.50 & 8.00 \\
\hline
\end{tabular}

Table 13: Bounds for standard deviation parameters.

\begin{tabular}{rrrrrr}
\hline & $\sigma_{E_{0}}[\mathrm{MPa}]$ & $\sigma_{y_{0 s}}[\mathrm{MPa}]$ & $\sigma_{y_{c s}}[\mathrm{MPa}]$ & $\sigma_{d_{c}}$ & $\sigma_{\omega}[\mathrm{MPa}]$ \\
\hline Lower bound & $8.00 \times 10^{3}$ & $5.00 \times 10^{-5}$ & 0.100 & 0.005 & 0.500 \\
\hline Upper bound & $1.80 \times 10^{4}$ & $1.00 \times 10^{-4}$ & 1.2 & 0.9 & 2.00 \\
\hline
\end{tabular}




\section{Exact and noisy data}

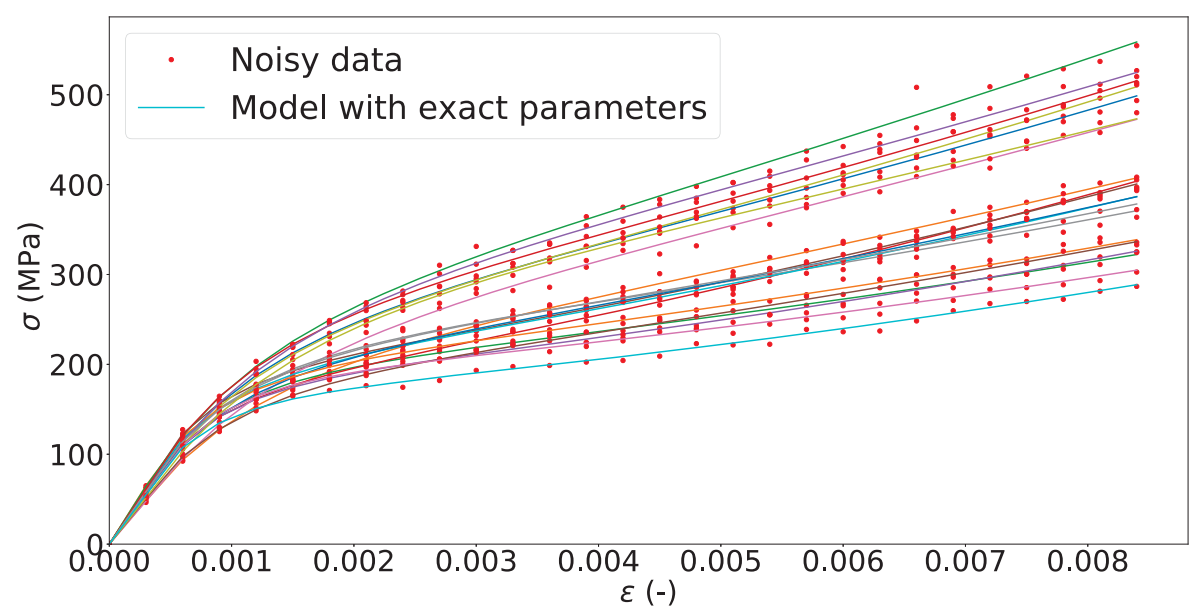

Figure 18: Model with exact individual parameters and noisy data. 


\section{E Results of calibration for all experiments from CERASEP A400 material}

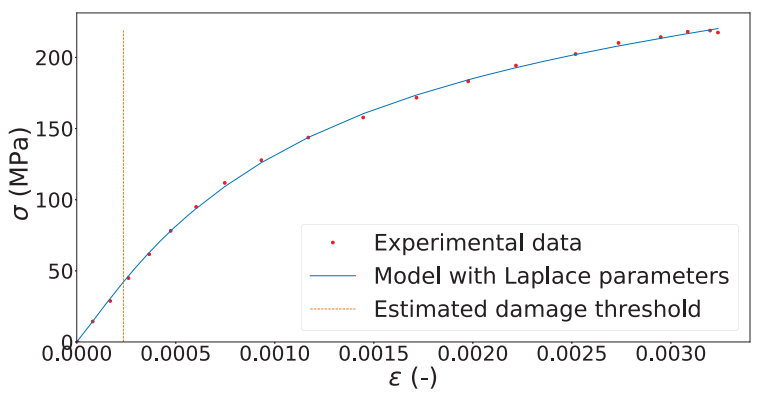

(a)

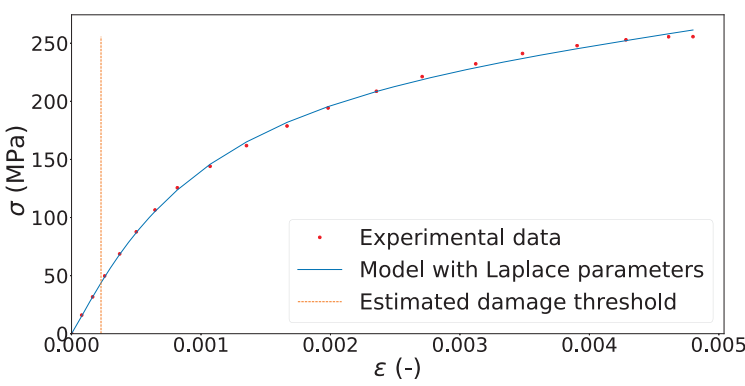

(c)

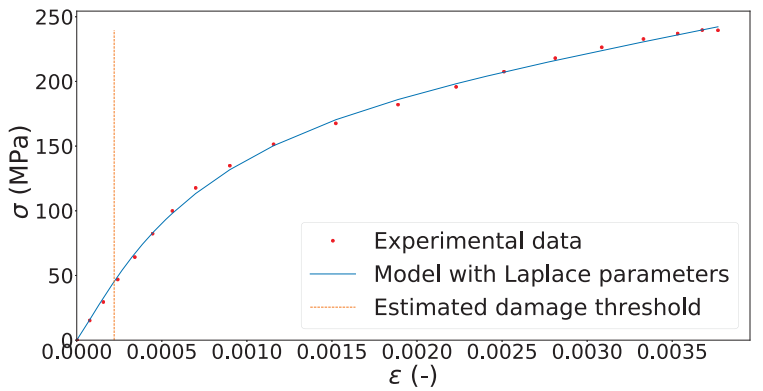

(e)

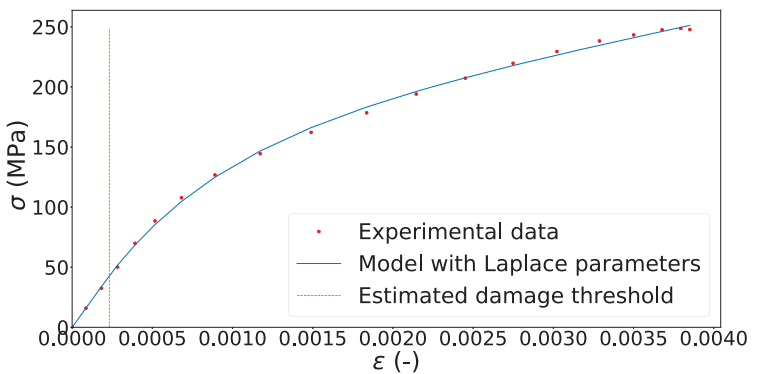

$(\mathrm{g})$

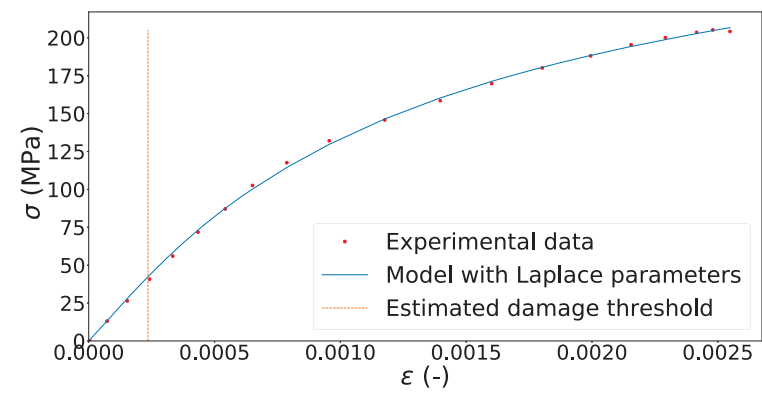

(b)

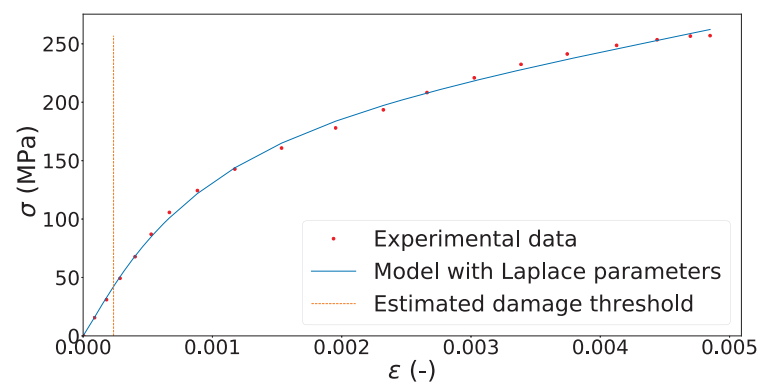

(d)

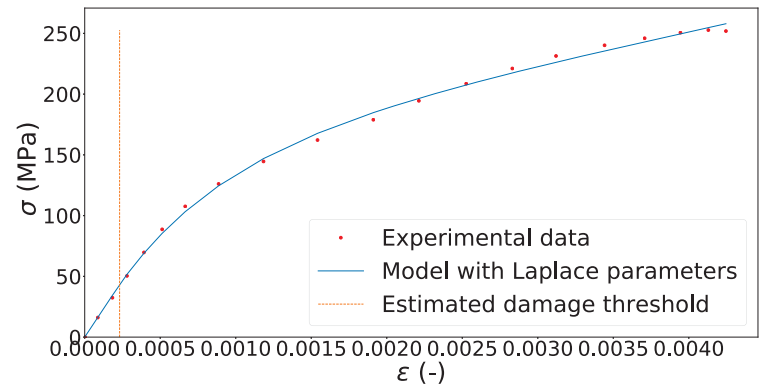

(f)

Figure 19: Model output vs experimental data for the first experiment (a), the second experiment (b), third experiment (c), fourth experiment (d), fifth experiment (e), sixth experiment (f) and the seventh experiment $(\mathrm{g})$. 


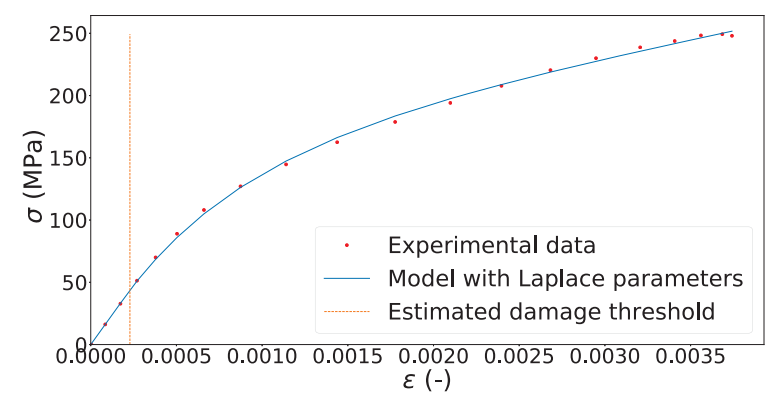

(a)

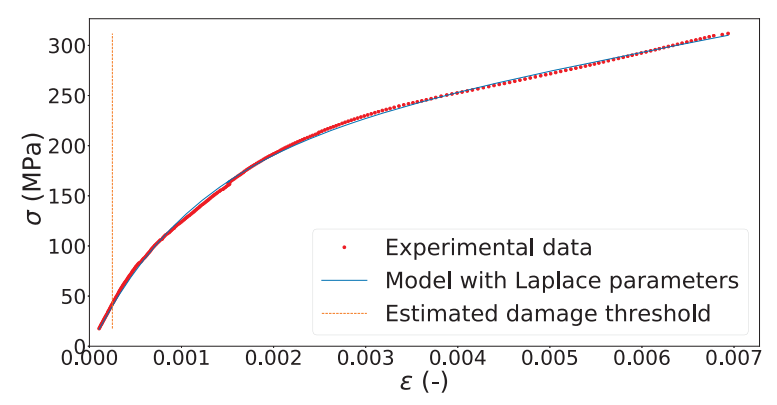

(c)

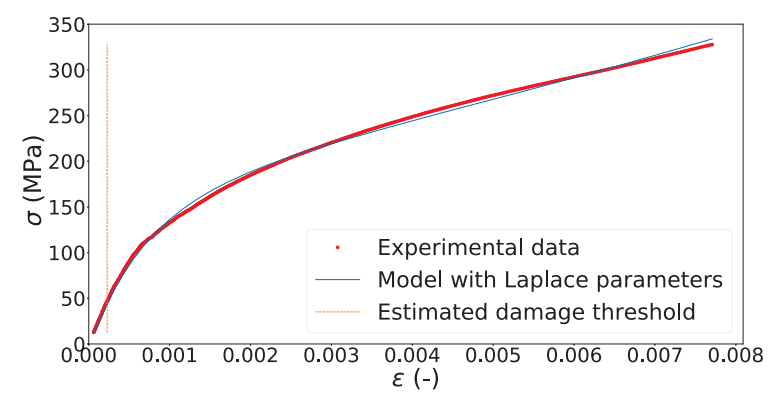

(e)

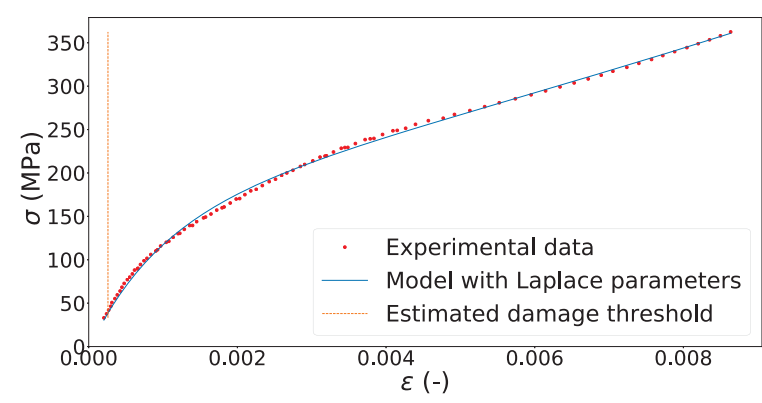

(b)

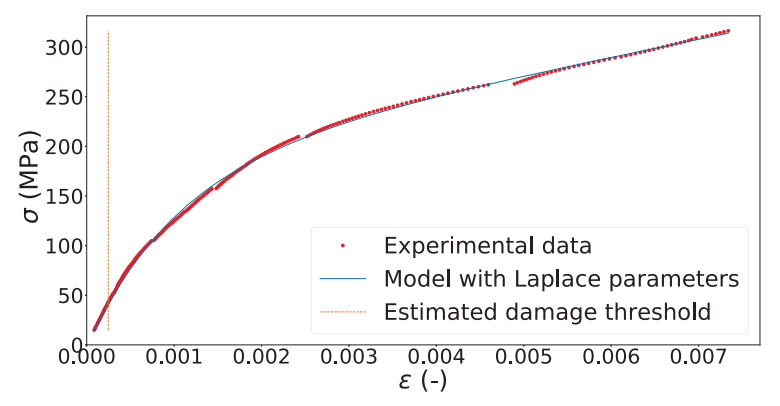

(d)

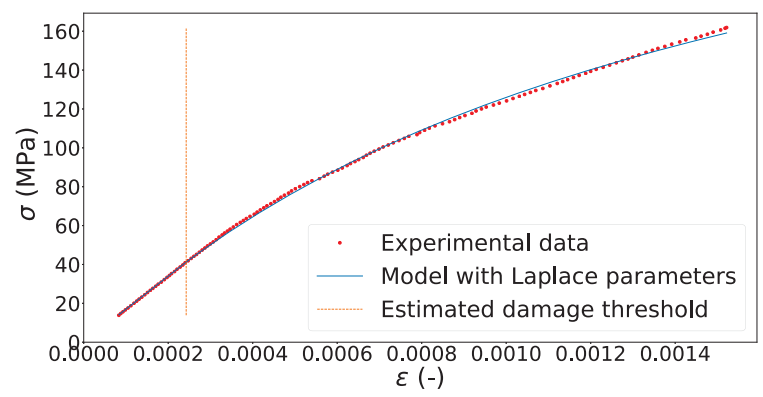

(f)

Figure 20: Model output vs experimental data for the eighth experiment (a), the ninth experiment (b), the tenth experiment (c), the eleventh experiment (d), the twelfth experiment (e), and the thirtheenth experiment (f). 


\section{REFERENCES}

[1] A. Efstratiadis and D. Koutsoyiannis, "One decade of multi-objective calibration approaches in hydrological modelling: a review.," Hydrological Sciences Journal, vol. 55, 2010.

[2] A. Anestis and C. René, Régression non linéaire et applications. (in french). Économie et statistiques avancées., Paris: Economica, 1992.

[3] E. Anane, D. C. Lpez C, T. Barz, G. Sin, K. V. Gernaey, P. Neubauer, and M. N. Cruz Bournazou, "Output uncertainty of dynamic growth models: effect of uncertain parameter estimates on model reliability.," Biochemical Engineering Journal, vol. 150, p. 107247, 2019.

[4] H. Rappel, L. Beex, J. Hale, L. Noels, and S. Bordas, "A tutorial on bayesian inference to identify material parameters in solid mechanics.," Archives of Computational Methods in Engineering, 2019.

[5] U. Defense, T. P. Company, M. S. Corporation, A. S. for Testing, and Materials, Composite Materials Handbook-MIL 17, Volume III: Materials Usage, Design, and Analysis. The Composite Materials Handbook-MIL 17, Taylor \& Francis, 1999.

[6] M. Gallagher and J. Doherty, "Parameter estimation and uncertainty analysis for a watershed model.," Environmental Modelling \& Software, vol. 22, no. 7, pp. 1000-1020, 2007.

[7] A. Tarantola, Inverse problem theory and methods for model parameter estimation. Society for Industrial and Applied Mathematics, 1 ed., 2005.

[8] M. C. Kennedy and A. O'Hagan, "Bayesian calibration of computer models.," Journal of the Royal Statistical Society: Series B (Statistical Methodology), vol. 63, no. 3, pp. 425464, 2001.

[9] L. Wasserman, All of Statistics: A Concise Course in Statistical Inference. Springer Publishing Company, Incorporated, 2010.

[10] M. Merriman, A List of Writings Relating to the Method of Least Squares: With Historical and Critical Notes. Transactions of the Connecticut Academy of Arts and Sciences, Academy, 1877.

[11] H. T. Banks, S. Hu, and W. C. Thompson, Modeling and Inverse Problems in the Presence of Uncertainty. Chapman and Hall/CRC, 2014.

[12] P. Janssen and P. Heuberger, "Calibration of process-oriented models.," Ecological Modelling, vol. 83, no. 1, pp. 55-66, 1995.

[13] R. Fisher and K. Pearson, On an Absolute Criterion for Fitting Frequency Curves. Messenger of mathematics, 1911.

[14] G. Young, "Mathematical statistics: An introduction to likelihood based inference.," International Statistical Review, vol. 87, pp. 178-179, 2019.

[15] J. A. Vrugt, H. V. Gupta, L. A. Bastidas, W. Bouten, and S. Sorooshian, "Effective and efficient algorithm for multiobjective optimization of hydrologic models: multiobjective optimization of hydrologic models.," vol. 39, no. 8, 2003. 
[16] Y. Collette and P. Siarry, Optimisation multiobjectif. (in french), vol. 44 of Algorithmes (Paris). Eyrolles, 2002.

[17] R. T. Bradley Efron, An introduction to bootstrap. Chapman \& Hall/CRC Monographs on Statistics \& Applied Probability, Chapman \& Hall, 1 ed., 1994.

[18] B. Efron, "Bootstrap methods: Another look at the jackknife.," The Annals of Statistics, vol. 7, no. 1, pp. 1-26, 1979.

[19] C. P. Robert and G. Casella, Monte Carlo Statistical Methods. Springer-Verlag, 2005.

[20] J. S. Liu, Monte Carlo Strategies in Scientific Computing, vol. 44. 2002.

[21] N. Metropolis, A. W. Rosenbluth, M. N. Rosenbluth, A. H. Teller, and E. Teller, "Equation of state calculations by fast computing machines.", The Journal of Chemical Physics, vol. 21, no. 6, pp. 1087-1092, 1953.

[22] W. K. Hastings, "Monte Carlo sampling methods using Markov Chains and their applications.," Biometrika, vol. 57, p. 14, 1970.

[23] S. Avril, M. Bonnet, A. S. Bretelle, M. Grédiac, F. Hild, P. Ienny, F. Latourte, D. Lemosse, S. Pagano, E. Pagnacco, and F. Pierron, "Overview of identification methods of mechanical parameters based on full-field measurements.," Experimental Mechanics, vol. 48, pp. 381-402, 2008.

[24] J. Isenberg, "Progressing from least squares to bayesian estimation.," Proc. of ASME Design Engineering Technical Conferences, 1979.

[25] W. Chongshuai, H. Yiqian, and Y. Haitian, "A SBFEM and sensitivity analysis based algorithm for solving inverse viscoelastic problems.," vol. 106, pp. 588-598, 2019.

[26] K. Solanki, M. Horstemeyer, W. Steele, Y. Hammi, and J. Jordon, "Calibration, validation, and verification including uncertainty of a physically motivated internal state variable plasticity and damage model.," International Journal of Solids and Structures, vol. 47, no. 2, pp. 186-203, 2010.

[27] C. Gogu, R. Haftka, R. Le Riche, J. Molimard, and A. Vautrin, "Introduction to the bayesian approach applied to elastic constants identification.," AIAA Journal, vol. 48, no. 5, pp. 893-903, 2010.

[28] C. Gogu, W. Yin, R. Haftka, P. Ifju, J. Molimard, R. L. Riche, and A. Vautrin, "Approche bayesienne pour grer les incertitudes dans lidentification partir de mesures de champ.(in french).," p. 6, 2011.

[29] P. Liu and S.-K. Au, "Bayesian parameter identification of hysteretic behavior of composite walls.," Probabilistic Engineering Mechanics, vol. 34, p. 101109, 2013.

[30] F. Rizzi, M. Khalil, R. E. Jones, J. A. Templeton, J. T. Ostien, and B. L. Boyce, "Bayesian modeling of inconsistent plastic response due to material variability.," p. 18, 2019.

[31] H. Rappel, L. A. A. Beex, and S. P. A. Bordas, "Bayesian inference to identify parameters in viscoelasticity.," vol. 22, no. 2, pp. 221-258, 2015. 
[32] M. Lavielle, Mixed Effects Models for the Population Approach: Models, Tasks, Methods and Tools. 2014.

[33] J.-F. Maire, P. M. Lesne, and R. Girard, "An explicit behavioural damage model for the design of components in ceramic matrix composites.," Key Engineering Materials, vol. 127131, pp. 1053-1060, 1997.

[34] L. Marcin, J.-F. Maire, N. Carrre, and E. Martin, "Development of a macroscopic damage model for woven ceramic matrix composites.," International Journal of Damage Mechanics, vol. 20, no. 6, pp. 939-957, 2011.

[35] C. B. Ramdane, "étude et modélisation du comportement mécanique de CMC oxyde/oxyde. (in french).," Thèse de doctorat de l' Université de Bordeaux I, 2014.

[36] R. Naslain, "Design, preparation and properties of non-oxide cmcs for application in engines and nuclear reactor: An overview," Composites Science and Technology, vol. 64, pp. 155-170, 2004.

[37] J. Viricelle, P. Goursat, and D. Bahloul-Hourlier, "Oxidation behaviour of a multi-layered ceramic-matrix composite (sic)f/c/(sibc)m.," Composites Science and Technology, vol. 61, no. 4, pp. 607-614, 2001.

[38] R. A. Fisher, "The correlation between relatives on the supposition of mendelian inheritance.," 1919.

[39] R. Drikvandi, "Nonlinear mixed-effects models for pharmacokinetic data analysis: assessment of the random-effects distribution.," Journal of Pharmacokinetics and Pharmacodynamics, vol. 44, 2017.

[40] C. R. Henderson, O. Kempthorne, S. R. Searle, and C. M. von Krosigk, "The estimation of environmental and genetic trends from records subject to culling.," Biometrics, vol. 15, no. 2, pp. 192-218, 1959.

[41] C. R. Henderson, "Best linear unbiased estimation and prediction under a selection model.," Biometrics, vol. 31, no. 2, pp. 423-447, 1975.

[42] N. M. Laird and J. H. Ware, "Random-effects models for longitudinal data.," Biometrics, vol. 38, no. 4, pp. 963-974, 1982.

[43] M. J. Lindstrom and D. M. Bates, "Nonlinear mixed effects models for repeated measures data.," Biometrics, vol. 46, no. 3, pp. 673-687, 1990.

[44] E. Demidenko, Mixed models. Theory and applications with R. 2nd ed. 2013.

[45] M. Davidian and D. Giltinan, Nonlinear Models for Repeated Measurement Data. Chapman \& Hall/CRC Monographs on Statistics \& Applied Probability, Taylor \& Francis, 1995.

[46] N. Metropolis and S. Ulam, “The Monte Carlo method.," Journal of the American Statistical Association, vol. 44, no. 247, pp. 335-341, 1949. 
[47] E. Kuhn and M. Lavielle, "Coupling a stochastic approximation version of em with a mcmc procedure.," Probability and Statistics, vol. 8, 2004.

[48] J. Pinheiro and D. Bates, Mixed-Effect Models in S and S-plus., vol. 96. 2002.

[49] M. Wand and M. Jones, Kernel Smoothing. Chapman \& Hall/CRC Monographs on Statistics \& Applied Probability, Taylor \& Francis, 1994.

[50] A. Azevedo-Filho and R. Shachter, "Laplace's method approximations for probabilistic inference in belief networks with continuous variables.," pp. 28-36, 1994.

[51] D. Bates, D. Hamilton, and D. Watts, "Calculation of intrinsic and parameter-effects curvatures for nonlinear regression models.," Communications in Statistics - Simulation and Computation, vol. 12, pp. 469-477, 1983.

[52] G. Stegmann, R. Jacobucci, J. Harring, and K. Grimm, "Nonlinear mixed-effects modeling programs in R.," Structural Equation Modeling: A Multidisciplinary Journal, vol. 25, pp. 1-6, 2017.

[53] E. Comets, A. P. Lavenu, and M. Lavielle, "Parameter estimation in nonlinear mixed effect models using saemix, an R implementation of the SAEM algorithm.," Journal of Statistical Software, vol. 80, no. 3, 2017.

[54] D. Packwood, Bayesian Optimization for Materials Science. SpringerBriefs in the Mathematics of Materials 3, Springer Singapore, 1 ed., 2017.

[55] M. D. McKay, R. J. Beckman, and W. J. Conover, "A comparison of three methods for selecting values of input variables in the analysis of output from a computer code.," Technometrics, vol. 21, no. 2, pp. 239-245, 1979.

[56] C. Rasmussen, O. Bousquet, U. Luxburg, and G. Rtsch, "Gaussian processes in machine learning.," Advanced Lectures on Machine Learning: ML Summer Schools 2003, Canberra, Australia, February 2 - 14, 2003, Tbingen, Germany, August 4 - 16, 2003, Revised Lectures, 63-71 (2004), vol. 3176, 2004.

[57] D. Jones, M. Schonlau, and W. Welch, "Efficient global optimization of expensive blackbox functions.," Journal of Global Optimization, vol. 13, pp. 455-492, 1998.

[58] S. Kullback and R. A. Leibler, "On information and sufficiency.," The Annals of Mathematical Statistics, vol. 22, no. 1, pp. 79 - 86, 1951.

[59] D. Foreman-Mackey, D. W. Hogg, D. Lang, and J. Goodman, "emcee: The mcmc hammer.," vol. 125, no. 925, p. 306, 2013.

[60] N. Hansen, S. Müller, and P. Koumoutsakos, "Reducing the time complexity of the derandomized evolution strategy with covariance matrix adaptation (CMA-ES).," Evolutionary computation, vol. 11, pp. 1-18, 2003.

[61] GPy, "A Gaussian process framework in python," 2012. 